\title{
Taylor collocation method for a system of nonlinear Volterra delay integro-differential equations with application to COVID-19 epidemic
}

Hafida Laib ( $\sim$ hafida.laib@gmail.com )

University Center Abdelhafd Boussouf, Mila, Algeria https://orcid.org/0000-0001-9935-9512

\section{Azzeddine Bellour}

Ecole Normale Supérieure de Constantine, Constantine, Algeria

Aissa Boulmerka

University Center Abdelhafd Boussouf, Mila, Algeria https://orcid.org/0000-0003-4920-1850

\section{Method Article}

Keywords: Nonlinear Volterra delay integro-differential equations, Collocation method, Taylor polynomials, Epidemic mathematical model, Corona virus

Posted Date: November 17th, 2020

DOI: https://doi.org/10.21203/rs.3.rs-107709/v1

License: (9) This work is licensed under a Creative Commons Attribution 4.0 International License. Read Full License

Version of Record: A version of this preprint was published at International Journal of Computer Mathematics on June 28th, 2021. See the published version at https://doi.org/10.1080/00207160.2021.1938012. 
Taylor collocation method for a system of nonlinear Volterra delay integro-differential equations with application to COVID-19 epidemic

Hafida Laib · Azzeddine Bellour · Aissa

Boulmerka

Received: date / Accepted: date

Abstract The present paper deals with the numerical solution for a general form of a system of nonlinear Volterra delay integro-differential equations (VDIDEs). The main purpose of this work is to provide a current numerical method based on the use of continuous collocation Taylor polynomials for the numerical solution of nonlinear VDIDEs systems. It is shown that this method is convergent. Numerical results will be presented to prove the validity and effectiveness of this convergent algorithm. We apply two models to the COVID-19 epidemic in China and one for the Predator-Prey model in mathematical ecology.

Keywords Nonlinear Volterra delay integro-differential equations · Collocation method · Taylor polynomials · Epidemic mathematical model · Corona virus

Mathematics Subject Classification (2010) 45G15 - 34K28 · 45D05 - 45L05 · $65 \mathrm{~L} 60$

H. Laib

Laboratory of Mathematics and their interactions, University Center Abdelhafid Boussouf, Mila, Algeria.

E-mail: hafida.laib@gmail.com

A. Bellour

Laboratory of Applied Mathematics and Didactics Ecole Normale Supérieure de Constantine, Constantine, Algeria.

E-mail: bellourazze123@yahoo.com

A. Boulmerka

Laboratory of Mathematics and their interactions, University Center Abdelhafid Boussouf, Mila, Algeria.

E-mail: aissaboulmerka@gmail.com 
1 Introduction

In this paper, a numerical method is presented to obtain an approximate solution for the following system of nonlinear delay integro-differential equations

$$
y^{\prime}(t)=f(t, y(t), y(t-\tau))+\int_{t-\tau}^{t} k(t, s, y(t), y(s)) d s,
$$

for $t \in[0, T]$ and $y(t)=\Phi(t)$ for $t \in[-\tau, 0]$ with $\Phi: \mathbb{R} \rightarrow \mathbb{R}^{d}$, where the functions $f: \mathbb{R} \times \mathbb{R}^{d} \times \mathbb{R}^{d} \rightarrow \mathbb{R}^{d}$ and $k: \mathbb{R} \times \mathbb{R} \times \mathbb{R}^{d} \times \mathbb{R}^{d} \rightarrow \mathbb{R}^{d}$ are sufficiently smooth.

The existence and the uniqueness of the solution of (1) can be found, for example, in $[10,11]$. The delay integro-differential equations and their systems have become important in the mathematical modeling of many fields of sciences and engineering (see, e.g., $[9,12,18,21,25,29,30])$. As a particular case in traditional population biology, The system of 'predator-prey' dynamics, which was first modeled by Volterra [27]. Moreover, Liu et al. [22] presents a COVID-19 epidemic model, which can be described by a particular form of the system of nonlinear delay integro-differential (1). We will present some applications of this system in Section 4 .

Recently, many numerical methods have been proposed to approximate the solution of system of nonlinear integro-differential equations. For example, operational Tau method [1], linear barycentric rational method [2], Adomian decomposition method [3], differential transform method [5], He's homotopy perturbation method [8], variational iteration method $([20,24,25])$, collocation method $[13-15,17,26]$.

The Taylor polynomial method for approximating the solution of integral equations and integro-differential equations has been proposed. Bellour and Bousselsal $[6,7]$ used Taylor collocation method for solving delay integral equations and integro-differential equations, Taylor collocation method for the Volterra Fredholm integral equations is used in [28], Gülsu and Sezer [16] applied a Taylor collocation method for the solution of systems of high-order Fredholm Volterra integro-differential equations.

The aim of this paper is to generalize the Taylor collocation method in [6] and [7] to construct an approximate solution for a general form of the system of nonlinear Volterra delay integro-differential equations (1). In our method, the approximate solution is explicit, direct, high order of convergence and obtained by using simple iterative formulas.

The paper is organized as follows: In section 2, we divide the interval $[0, T]$ into subintervals, and we approximate the solution of (1) in each interval by a Taylor polynomial. The convergence analysis is established in section 3 , and the numerical illustrations are provided in section 4.

\section{Description of the Method}

We suppose that $T=r \tau$, where $r \in\{1,2,3, \ldots\}$. Let $\Pi_{N}$ be a uniform partition of the interval $I=[0, T]$ defined by $t_{n}^{i}=i \tau+n h, n=0,1, . ., N, i=0,1, . ., r-1$, 
where the stepsize is given by $h=\frac{\tau}{N}$. Define the subintervals $\sigma_{n}^{i}=\left[t_{n}^{i} ; t_{n+1}^{i}\right), n=$ $0,1, . ., N-1, i=0,1, . ., r-1$ and $\sigma_{N-1}^{r-1}=\left[t_{N-1}^{r-1}, t_{N}^{r-1}\right]$. Moreover, denote by $\pi_{m}$ the set of all real polynomials of degree not exceeding $m$. We define the real polynomial spline space of degree $m$ as follows:

$$
S_{m}^{(0)}\left(\Pi_{N}\right)=\left\{u \in C\left(I, \mathbb{R}^{d}\right): u_{n}^{i}=\left.u\right|_{\sigma_{n}^{i}} \in \pi_{m}, n=0, \ldots, N-1, i=0,1, . ., r-1\right\}
$$

This is the space of piecewise polynomials of degree (at most) $m$. Its dimension is $r N m+1$, i.e., the same as the total number of the coefficients of the polynomials $u_{n}^{i}, n=0, \ldots, N-1, i=0,1, . ., r-1$. To find these coefficients, we use Taylor polynomial on each subinterval.

First, we approximate the exact solution $y$ in the interval $\sigma_{0}^{0}$ by the polynomial

$$
u_{0}^{0}(t)=\sum_{j=0}^{m} \frac{y^{(j)}(0)}{j !} t^{j} ; \quad t \in \sigma_{0}^{0}
$$

To find $y^{(j)}(0)$, we differentiate equation (1) $j$-times, we obtain

$$
\begin{aligned}
y^{(j+1)}(0)=f^{(j)}(0, \Phi(0), \Phi(-\tau)) & +\left(\int_{t-\tau}^{0} k(t, s, \Phi(t), \Phi(s)) d s\right)^{(j)}(0) \\
& +\sum_{i=0}^{j-1}\left[\partial_{t}^{(i)} k(t, t, y(t), y(t))\right]^{(j-1-i)}(0),
\end{aligned}
$$

for $j=0,1, \ldots, m$, where $y(0)=\Phi(0)$.

Second, we approximate $y$ by the polynomial $u_{n}^{0}(n \in\{1,2, \ldots, N-1\})$ on the interval $\sigma_{n}^{0}$ such that

$$
u_{n}^{0}(t)=\sum_{j=0}^{m} \frac{\hat{u}_{n, 0}^{(j)}\left(t_{n}^{0}\right)}{j !}\left(t-t_{n}^{0}\right)^{j} ; \quad t \in \sigma_{n}^{0}
$$

where $\hat{u}_{n, 0}$ is the exact solution of the integro-differential equation

$$
\begin{aligned}
\hat{u}_{n, 0}^{\prime}(t)= & f\left(t, u_{n-1}^{0}(t), \Phi(t-\tau)\right)+\int_{t-\tau}^{0} k(t, s, \Phi(t), \Phi(s)) d s \\
& +\sum_{i=0}^{n-1} \int_{t_{i}^{0}}^{t_{i+1}^{0}} k\left(t, s, u_{i}^{0}(t), u_{i}^{0}(s)\right) d s+\int_{t_{n}^{0}}^{t} k\left(t, s, \hat{u}_{n, 0}(t), \hat{u}_{n, 0}(s)\right) d s,
\end{aligned}
$$


for $t \in \sigma_{n}^{0}$ such that $\hat{u}_{n, 0}\left(t_{n}^{0}\right)=u_{n-1}^{0}\left(t_{n}^{0}\right)$.

The coefficients $\hat{u}_{n, 0}^{(j)}\left(t_{n}^{0}\right)$ are given by the following formula

$$
\begin{aligned}
\hat{u}_{n, 0}^{(j+1)}\left(t_{n}^{0}\right)= & f^{(j)}\left(t_{n}^{0}, u_{n-1}^{0}\left(t_{n}^{0}\right), \Phi\left(\left(t_{n}^{0}-\tau\right)\right)+\left(\int_{t-\tau}^{0} k(t, s, \Phi(t), \Phi(s)) d s\right)^{(j)}\left(t_{n}^{0}\right)\right. \\
& +\sum_{i=0}^{j-1}\left[\partial_{t}^{(i)} k\left(t, t, \hat{u}_{n, 0}(t), \hat{u}_{n, 0}(t)\right)\right]^{(j-1-i)}\left(t_{n}^{0}\right) \\
& +\sum_{i=0}^{n-1} \int_{t_{i}^{0}}^{t_{i+1}^{0}} \partial_{t}^{(j)} k\left(t_{n}^{0}, s, u_{i}^{0}\left(t_{n}^{0}\right), u_{i}^{0}(s)\right) d s
\end{aligned}
$$

for $j \in\{0,1, \ldots, m\}$ and $\hat{u}_{n, 0}\left(t_{n}^{0}\right)=u_{n-1}^{0}\left(t_{n}^{0}\right)$.

Third, for $y$ to be approximated by $u_{0}^{p}(p \in\{1,2, \ldots, r-1\})$ on the interval $\sigma_{0}^{p}$, $y$ is to be approximated by $u_{k}^{j}(0 \leq k \leq N-1$ and $0 \leq j<p)$ on each interval $\sigma_{k}^{j}$ such that

$$
u_{0}^{p}(t)=\sum_{j=0}^{m} \frac{\hat{u}_{0, p}^{(j)}\left(t_{0}^{p}\right)}{j !}\left(t-t_{0}^{p}\right)^{j} ; \quad t \in \sigma_{0}^{p},
$$

where $\hat{u}_{0, p}$ is the exact solution of the integro-differential equation

$$
\begin{aligned}
\hat{u}_{0, p}^{\prime}(t)=f\left(t, u_{N-1}^{p-1}(t), u_{0}^{p-1}(t-\tau)\right) & +\int_{t-\tau}^{t_{0}^{p}} k\left(t, s, u^{p-1}(t), u^{p-1}(s)\right) d s \\
& +\int_{t_{0}^{p}}^{t} k\left(t, s, \hat{u}_{0, p}(t), \hat{u}_{0, p}(s)\right) d s,
\end{aligned}
$$

for $t \in \sigma_{0}^{p}$ such that $\hat{u}_{0, p}\left(t_{0}^{p}\right)=u_{N-1}^{p-1}\left(t_{0}^{p}\right)$, where $u^{p-1}=u$ on the interval $\sigma^{p-1}=$ $\left[t_{0}^{p-1}, t_{0}^{p}\right]$ for $p \in\{0, \ldots, r\}$.

The coefficients $\hat{u}_{0, p}^{(j)}\left(t_{0}^{p}\right)$ are given by the following formula

$$
\begin{aligned}
\hat{u}_{0, p}^{(j+1)}\left(t_{0}^{p}\right)= & f^{(j)}\left(t_{0}^{p}, u_{N-1}^{p-1}\left(t_{0}^{p}\right), u_{0}^{p-1}\left(t_{0}^{p}-\tau\right)\right) \\
& -\sum_{i=0}^{j-1}\left[\partial_{t}^{(i)} k\left(t, t-\tau, u_{0}^{p-1}(t), u_{0}^{p-1}(t-\tau)\right)\right]^{(j-1-i)}\left(t_{0}^{p}\right) \\
& +\sum_{i=0}^{N-1} \int_{t_{i}^{p-1}}^{t_{i+1}^{p-1}} \partial_{t}^{(j)} k\left(t_{0}^{p}, s, u_{i}^{p-1}\left(t_{0}^{p}\right), u_{i}^{p-1}(s)\right) d s \\
& +\sum_{i=0}^{j-1}\left[\partial_{t}^{(i)} k\left(t, t, \hat{u}_{0, p}(t), \hat{u}_{0, p}(t)\right)\right]^{(j-1-i)}\left(t_{0}^{p}\right),
\end{aligned}
$$

for $j \in\{0,1, \ldots, m\}$ and $\hat{u}_{0, p}\left(t_{0}^{p}\right)=u_{N-1}^{p-1}\left(t_{0}^{p}\right)$.

Finally, on the interval $\sigma_{n}^{p}(n \in\{1,2, \ldots, N-1\})$, the polynomial $u_{n}^{p}$ is defined by the following formula

$$
u_{n}^{p}(t)=\sum_{j=0}^{m} \frac{\hat{u}_{n, p}^{(j)}\left(t_{n}^{p}\right)}{j !}\left(t-t_{n}^{p}\right)^{j} ; \quad t \in \sigma_{n}^{p}
$$


where $\hat{u}_{n, p}$ is the exact solution of the integro-differential equation

$$
\begin{aligned}
\hat{u}_{n, p}^{\prime}(t)= & f\left(t, u_{n-1}^{p}(t), u_{n}^{p-1}(t-\tau)\right)+\int_{t-\tau}^{t_{0}^{p}} k\left(t, s, u^{p-1}(t), u^{p-1}(s)\right) d s \\
& +\sum_{i=0}^{n-1} \int_{t_{i}^{p}}^{t_{i+1}^{p}} k\left(t, s, u_{i}^{p}(t), u_{i}^{p}(s)\right) d s+\int_{t_{n}^{p}}^{t} k\left(t, s, \hat{u}_{n, p}(t), \hat{u}_{n, p}(s)\right) d s,
\end{aligned}
$$

for $t \in \sigma_{n}^{p}$ such that $\hat{u}_{n, p}\left(t_{n}^{p}\right)=u_{n-1}^{p}\left(t_{n}^{p}\right)$.

The coefficients $\hat{u}_{n, p}^{(j)}\left(t_{n}^{p}\right)$ are given by the following formula

$$
\begin{aligned}
\hat{u}_{n, p}^{(j+1)}\left(t_{n}^{p}\right)= & f^{(j)}\left(t_{n}^{p}, u_{n-1}^{p}\left(t_{n}^{p}\right), u_{n}^{p-1}\left(t_{n}^{p}-\tau\right)\right) \\
& -\sum_{i=0}^{j-1}\left[\partial_{t}^{(i)} k\left(t, t-\tau, u_{n}^{p-1}(t), u_{n}^{p-1}(t-\tau)\right)\right]^{(j-1-i)}\left(t_{n}^{p}\right) \\
& +\sum_{i=0}^{N-1} \int_{t_{i}^{p-1}}^{t_{i+1}^{p-1}} \partial_{t}^{(j)} k\left(t_{n}^{p}, s, u_{i}^{p-1}\left(t_{n}^{p}\right), u_{i}^{p-1}(s)\right) d s \\
& +\sum_{i=0}^{j-1}\left[\partial_{t}^{(i)} k\left(t, t, \hat{u}_{n, p}(t), \hat{u}_{n, p}(t)\right)\right]^{(j-1-i)}\left(t_{n}^{p}\right) \\
& +\sum_{i=0}^{n-1} \int_{t_{i}^{p}}^{t_{i+1}^{p}} \partial_{t}^{(j)} k\left(t_{n}^{p}, s, u_{i}^{p}\left(t_{n}^{p}\right), u_{i}^{p}(s)\right) d s,
\end{aligned}
$$

for $j \in\{0,1, \ldots, m\}$ and $\hat{u}_{n, p}\left(t_{n}^{p}\right)=u_{n-1}^{p}\left(t_{n}^{p}\right)$.

\section{Analysis of Convergence}

For ease of exposition, we will consider a feasible linear form of (1), namely

$$
y^{\prime}(t)=g(t)+a(t) y(t)+b(t) y(t-\tau)+\int_{t-\tau}^{t} k(t, s) y(s) d s,
$$

More precisely, the equations (4), (5), (7), (8), (10) and (11) may be written in the following linear forms, respectively,

$$
\begin{aligned}
\hat{u}_{n, 0}^{\prime}(t)=g(t) & +a(t) \hat{u}_{n, 0}(t)+b(t) \Phi(t-\tau)+\int_{t-\tau}^{0} k(t, s) \Phi(s) d s \\
& +\sum_{i=0}^{n-1} \int_{t_{i}^{0}}^{t_{i+1}^{0}} k(t, s) u_{i}^{0}(s) d s+\int_{t_{n}^{0}}^{t} k(t, s) \hat{u}_{n, 0}(s) d s
\end{aligned}
$$




$$
\begin{aligned}
\hat{u}_{n, 0}^{(j+1)}\left(t_{n}^{0}\right)=f^{(j)}\left(t_{n}^{0}\right) & +\sum_{l=0}^{j}\left(\begin{array}{c}
j \\
l
\end{array}\right)\left(a^{(j-l)}\left(t_{n}^{0}\right) \hat{u}_{n, 0}^{(l)}\left(t_{n}^{0}\right)+b^{(j-l)}\left(t_{n}^{0}\right) \Phi^{(l)}\left(t_{n}^{0}-\tau\right)\right) \\
& +\left(\int_{t-\tau}^{0} k(t, s) \Phi(s) d s\right)^{(j)}\left(t_{n}^{0}\right)+\sum_{i=0}^{j-1}\left[\partial_{t}^{(i)} k(t, t) \hat{u}_{n, 0}(t)\right]^{(j-1-i)}\left(t_{n}^{0}\right) \\
& +\sum_{i=0}^{n-1} \int_{t_{i}^{0}}^{t_{i+1}^{0}} \partial_{t}^{(j)} k\left(t_{n}^{0}, s\right) u_{i}^{0}(s) d s \\
=f^{(j)}\left(t_{n}^{0}\right)+ & +\sum_{l=0}^{j}\left(\begin{array}{c}
j \\
l
\end{array}\right)\left(a^{(j-l)}\left(t_{n}^{0}\right) \hat{u}_{n, 0}^{(l)}\left(t_{n}^{0}\right)+b^{(j-l)}\left(t_{n}^{0}\right) \Phi^{(l)}\left(t_{n}^{0}-\tau\right)\right) \\
& +\left(\int_{t-\tau}^{0} k(t, s) \Phi(s) d s\right)^{(j)}\left(t_{n}^{0}\right) \\
& +\sum_{i=0}^{j-1} \sum_{l=0}^{j-1-i}\left(\begin{array}{c}
j-1-i \\
l
\end{array}\right)\left[\partial_{t}^{(i)} k(t, t)\right]^{(j-1-i-l)}\left(t_{n}^{0}\right) \hat{u}_{n, 0}^{(l)}\left(t_{n}^{0}\right) \\
& +\sum_{i=0}^{n-1} \sum_{l=0}^{m} \frac{\hat{u}_{i, 0}^{(l)}\left(t_{i}^{0}\right)}{l !} \int_{t_{i}^{0}}^{t_{i+1}^{0}} \partial_{t}^{(j)} k\left(t_{n}^{0}, s\right)\left(s-t_{i}^{0}\right)^{l} d s,
\end{aligned}
$$

$$
\begin{aligned}
\hat{u}_{0, p}^{\prime}(t)=g(t)+a(t) \hat{u}_{0, p}(t)+ & b(t) \hat{u}_{0, p-1}(t-\tau)+\int_{t-\tau}^{t_{0}^{p}} k(t, s) u^{p-1}(s) d s \\
& +\int_{t_{0}^{p}}^{t} k(t, s) \hat{u}_{0, p}(s) d s,
\end{aligned}
$$

$$
\begin{aligned}
\hat{u}_{0, p}^{(j+1)}\left(t_{0}^{p}\right)=f^{(j)}\left(t_{0}^{p}\right) & \left.+\sum_{l=0}^{j}\left(\begin{array}{c}
j \\
l
\end{array}\right)\left(a^{(j-l)}\left(t_{0}^{p}\right) \hat{u}_{0, p}^{(l)}\left(t_{0}^{p}\right)+b^{(j-l)}\left(t_{0}^{p}\right)\right) \hat{u}_{0, p-1}^{(l)}\left(t_{0}^{p-1}\right)\right) \\
& -\sum_{i=0}^{j-1} \sum_{l=0}^{j-1-i}\left(\begin{array}{c}
j-1-i \\
l
\end{array}\right)\left[\partial_{t}^{(i)} k(t, t-\tau)\right]^{(j-1-i-l)}\left(t_{0}^{p}\right) \hat{u}_{0, p-1}^{(l)}\left(t_{0}^{p-1}\right) \\
& +\sum_{i=0}^{N-1} \sum_{l=0}^{m} \frac{\hat{u}_{i, p-1}^{(l)}\left(t_{i}^{p-1}\right)}{l !} \int_{t_{i}^{p-1}}^{t_{i+1}^{p-1}} \partial_{t}^{(j)} k\left(t_{0}^{p}, s\right)\left(s-t_{i}^{p-1}\right)^{l} d s \\
& +\sum_{i=0}^{j-1} \sum_{l=0}^{j-1-i}\left(\begin{array}{c}
j-1-i \\
l
\end{array}\right)\left[\partial_{t}^{(i)} k(t, t)\right]^{(j-1-i-l)}\left(t_{0}^{p}\right) \hat{u}_{0, p}^{(l)}\left(t_{0}^{p}\right),
\end{aligned}
$$

$$
\begin{aligned}
\hat{u}_{n, p}^{\prime}(t)=g(t) & +a(t) \hat{u}_{n, p}(t)+b(t) \hat{u}_{n, p-1}(t-\tau)+\int_{t-\tau}^{t_{0}^{p}} k(t, s) u^{p-1}(s) d s \\
& +\sum_{i=0}^{n-1} \int_{t_{i}^{p}}^{t_{i+1}^{p}} k(t, s) u_{i}^{p}(s) d s+\int_{t_{n}^{p}}^{t} k(t, s) \hat{u}_{n, p}(s) d s,
\end{aligned}
$$


and

$$
\begin{aligned}
\hat{u}_{n, p}^{(j+1)}\left(t_{n}^{p}\right)=f^{(j)}\left(t_{n}^{p}\right) & \left.+\sum_{l=0}^{j}\left(\begin{array}{c}
j \\
l
\end{array}\right)\left(a^{(j-l)}\left(t_{n}^{p}\right) \hat{u}_{n, p}^{(l)}\left(t_{n}^{p}\right)+b^{(j-l)}\left(t_{n}^{p}\right)\right) \hat{u}_{n, p-1}^{(l)}\left(t_{n}^{p-1}\right)\right) \\
& -\sum_{i=0}^{j-1} \sum_{l=0}^{j-1-i}\left(\begin{array}{c}
j-1-i \\
l
\end{array}\right)\left[\partial_{t}^{(i)} k(t, t-\tau)\right]^{(j-1-i-l)}\left(t_{n}^{p}\right) \hat{u}_{n, p-1}^{(l)}\left(t_{n}^{p-1}\right) \\
& +\sum_{i=n}^{N-1} \sum_{l=0}^{m} \frac{\hat{u}_{i, p-1}^{(l)}\left(t_{i}^{p-1}\right)}{l !} \int_{t_{i}^{p-1}}^{t_{i+1}^{p-1}} \partial_{t}^{(j)} k\left(t_{n}^{p}, s\right)\left(s-t_{i}^{p-1}\right)^{l} d s \\
+ & \sum_{i=0}^{j-1} \sum_{l=0}^{j-1-i}\left(\begin{array}{c}
j-1-i \\
l
\end{array}\right)\left[\partial_{t}^{(i)} k(t, t)\right]^{(j-1-i-l)}\left(t_{n}^{p}\right) \hat{u}_{n, p}^{(l)}\left(t_{n}^{p}\right) \\
+ & \sum_{i=0}^{n-1} \sum_{l=0}^{m} \frac{\hat{u}_{i, p}^{(l)}\left(t_{i}^{p}\right)}{l !} \int_{t_{i}^{p}}^{t_{i+1}^{p}} \partial_{t}^{(j)} k\left(t_{n}^{p}, s\right)\left(s-t_{i}^{p}\right)^{l} d s,
\end{aligned}
$$

The following three lemmas will be used in this section.

Lemma 1 (Discrete Gronwall-type inequality [10]) Let $\left\{k_{j}\right\}_{j=0}^{n}$ be a given nonnegative sequence and the sequence $\left\{\varepsilon_{n}\right\}$ satisfies $\varepsilon_{0} \leq p_{0}$ and

$$
\varepsilon_{n} \leq p_{0}+\sum_{i=0}^{n-1} k_{i} \varepsilon_{i}, \quad n \geq 1
$$

with $p_{0} \geq 0$.Then $\varepsilon_{n}$ can be bounded by

$$
\varepsilon_{n} \leq p_{0} \exp \left(\sum_{j=0}^{n-1} k_{j}\right), \quad n \geq 1 .
$$

Lemma 2 (Discrete Gronwall-type inequality [4]) If $\left\{f_{n}\right\}_{n \geq 0},\left\{g_{n}\right\}_{n \geq 0}$ and $\left\{\varepsilon_{n}\right\}_{n \geq 0}$ are nonnegative sequences and

$$
\varepsilon_{n} \leq f_{n}+\sum_{i=0}^{n-1} g_{i} \varepsilon_{i}, \quad n \geq 0
$$

Then,

$$
\varepsilon_{n} \leq f_{n}+\sum_{i=0}^{n-1} f_{i} g_{i} \exp \left(\sum_{k=0}^{n-1} g_{k}\right), \quad n \geq 0 .
$$

Lemma 3 [19] Assume that the sequence $\left\{\varepsilon_{n}\right\}_{n \geq 0}$ of nonnegative numbers satisfies

$$
\varepsilon_{n} \leq A \varepsilon_{n-1}+B \sum_{i=0}^{n-1} \varepsilon_{i}+K, \quad n \geq 1,
$$

where $A, B$ and $K$ are nonnegative constants, then

$$
\varepsilon_{n} \leq \frac{\varepsilon_{0}}{R_{2}-R_{1}}\left[\left(R_{2}-1\right) R_{2}^{n}+\left(1-R_{1}\right) R_{1}^{n}\right]+\frac{K}{R_{2}-R_{1}}\left[R_{2}^{n}-R_{1}^{n}\right],
$$


where

$$
\begin{aligned}
& R_{1}=\left(1+A+B-\sqrt{(1-A)^{2}+B^{2}+2 A B+2 B}\right) / 2, \\
& R_{2}=\left(1+A+B+\sqrt{(1-A)^{2}+B^{2}+2 A B+2 B}\right) / 2,
\end{aligned}
$$

therefore, $0 \leq R_{1} \leq 1 \leq R_{2}$.

The next lemma will be crucial for establishing the convergence of the approximate solution. by

In the following, for a given fonction $\psi \in C\left(I, \mathbb{R}^{d}\right)$, we define the norm $\|\psi\|$

$$
\|\psi\|=\left\{\max \left|\psi_{i}(t)\right|, \quad t \in I, \quad i=1, \ldots, d\right\}
$$

Lemma 4 Let $g, k, a, b$ and $\Phi$ be $m$ times continuously differentiable on their respective domains. Then, there exists a positive number $\alpha(m)$ such that for all $n=0,1, \ldots, N-1, p=0,1, \ldots, r-1$ and $j=0,1, \ldots, m+1$, we have,

$$
\left\|\hat{u}_{n, p}^{(j)}\right\| \leq \alpha(m)
$$

provided that $h$ is sufficiently small, where $\hat{u}_{0,0}(t)=y(t)$ for $t \in \sigma_{0}^{0}$.

Proof. The proof is split into two steps.

Claim 1. There exists a positive constant $\alpha_{1}(m)$ such that $\left\|\hat{u}_{n, 0}^{(j)}\right\| \leq \alpha_{1}(m)$.

Let $a_{n}^{j}=\left\|\hat{u}_{n, 0}^{(j)}\right\|$, we have for all $j=0,1, \ldots, m+1$,

$$
a_{0}^{j} \leq \max \left\{\left\|y^{(j)}\right\|, j=0,1, \ldots, m+1\right\}=\alpha_{1}^{1}(m) .
$$

On the other hand, for $n \geq 1$, by differentiating equation (13) $j$-times, we obtain, for all $j=0,1, \ldots, m$,

$$
a_{n}^{j+1} \leq c_{1}+A \sum_{k=0}^{j} a_{n}^{k}+\left(m b_{1}^{1}+b_{1}^{2}\right) \sum_{k=0}^{j-1} a_{n}^{k}+h d_{1} \sum_{i=0}^{n-1} \sum_{k=0}^{m} a_{i}^{k},
$$

where

$$
\begin{gathered}
A=\max \left\{\left(\begin{array}{l}
j \\
l
\end{array}\right)\left\|a^{(j-l)}\right\|, l=0, \ldots, j ; \quad j=0, \ldots, m\right\} \\
c_{1}=\max \left\{\left\|g^{(j)}+\left(\int_{t-\tau}^{0} k(t, s) \Phi(s) d s\right)^{(j)}+(b(t) \Phi(t-\tau))(j)\right\|, j=0, \ldots, m\right\}, \\
b_{1}^{1}=\max \left\{\left(\begin{array}{c}
j-1-i \\
l
\end{array}\right)\left\|\left[\partial_{t}^{(i)} k(t, t)\right]^{(j-1-i-l)}\right\| ; j=1, \ldots, m ;\right. \\
i=0, \ldots, j-1 ; l=0, \ldots, j-1-i\}, \\
b_{1}^{2}=\max \left\{\left\|\int_{0}^{t} \partial_{t}^{(j)} k(t, s) d s\right\|, j=0, \ldots, m\right\},
\end{gathered}
$$

and

$$
d_{1}=\max \left\{\frac{1}{l !}\left\|\partial_{t}^{(j)} k(t, s)(s-v)^{l}\right\| ; \quad j=0, \ldots, m ; l=0, \ldots, m\right\}
$$


which implies that, for all $j \geq 1$,

$$
\begin{aligned}
a_{n}^{j} & \leq c_{1}+A \sum_{k=0}^{j-1} a_{n}^{k}+\left(m b_{1}+b_{1}^{2}\right) \sum_{k=0}^{j-2} a_{n}^{k}+h d_{1} \sum_{i=0}^{n-1} \sum_{k=0}^{m} a_{i}^{k} \\
& \leq c_{1}+\underbrace{\left(A+m b_{1}^{1}+b_{1}^{2}\right)}_{b_{1}} \sum_{k=0}^{j-1} a_{n}^{k}+h d_{1} \sum_{i=0}^{n-1} \sum_{k=0}^{m} a_{i}^{k} \\
& \leq c_{1}+b_{1} a_{n}^{0}+b_{1} \sum_{k=1}^{j-1} a_{n}^{k}+h d_{1} \sum_{i=0}^{n-1} \sum_{k=0}^{m} a_{i}^{k} .
\end{aligned}
$$

Now, for each fixed $n \geq 1$, we consider the sequence $y_{j}=a_{n}^{j}$ for $j=1,2, \ldots, m+1$. Then by $(20)$, the sequence $\left(y_{j}\right)$ satisfies for all $j=1,2, \ldots, m+1$

$$
y_{j} \leq c_{1}+b_{1} a_{n}^{0}+b_{1} \sum_{k=1}^{j-1} y_{k}+h d_{1} \sum_{i=0}^{n-1} \sum_{k=0}^{m} a_{i}^{k},
$$

hence, by Lemma 1 , for all $j=1,2, \ldots, m+1$

$$
\begin{aligned}
y_{j} & \leq \underbrace{c_{1} \exp \left(b_{1} m\right)}_{c_{2}}+\underbrace{b_{1} \exp \left(b_{1} m\right)}_{b_{2}} a_{n}^{0}+h \underbrace{d_{1} \exp \left(b_{1} m\right)}_{d_{2}} \sum_{i=0}^{n-1} \sum_{k=0}^{m} a_{i}^{k} \\
& \leq c_{2}+b_{2} a_{n}^{0}+h d_{2} \sum_{i=0}^{n-1} \sum_{k=0}^{m+1} a_{i}^{k} .
\end{aligned}
$$

Consider the sequence $z_{n}=\sum_{j=1}^{m+1} y_{j}=\sum_{j=1}^{m+1} a_{n}^{j}$ for $n \geq 0$.

Then by $(21)$, the sequence $\left(z_{n}\right)$ satisfies

$$
\begin{aligned}
z_{n} & \leq \underbrace{(m+1) c_{2}}_{c_{3}^{1}}+\underbrace{(m+1) b_{2}}_{b_{3}} a_{n}^{0}+h \underbrace{(m+1) d_{2}}_{d_{3}} \sum_{i=0}^{n-1} \sum_{k=0}^{m+1} a_{i}^{k} \\
& \leq c_{3}^{1}+b_{3} a_{n}^{0}+h d_{3} \sum_{i=0}^{n-1} a_{i}^{0}+h d_{3} \sum_{i=0}^{n-1} \sum_{k=1}^{m+1} a_{i}^{k} \\
& \leq c_{3}^{1}+b_{3} a_{n}^{0}+h d_{3} \sum_{i=0}^{n} a_{i}^{0}+h d_{3} \sum_{i=0}^{n-1} z_{i} .
\end{aligned}
$$

Moreover, from (19), we obtain,

$$
z_{0} \leq(m+2) \alpha_{1}^{1}(m)=c_{3}^{2} .
$$

Let $c_{3}=\max \left(c_{3}^{1}, c_{3}^{2}\right)$, then from (22) and (23), we get for all $n \geq 0$

$$
z_{n} \leq c_{3}+b_{3} a_{n}^{0}+h d_{3} \sum_{i=0}^{n} a_{i}^{0}+h d_{3} \sum_{i=0}^{n-1} z_{i} .
$$


Then, by Lemma 2, we obtain

$$
\begin{aligned}
z_{n} & \leq c_{3}+b_{3} a_{n}^{0}+h d_{3} \sum_{i=0}^{n} a_{i}^{0}+\sum_{j=0}^{n-1} h d_{3}\left(c_{3}+b_{3} a_{j}^{0}+h d_{3} \sum_{i=0}^{j} a_{i}^{0}\right) \exp \left(\tau d_{3}\right) \\
& \leq \underbrace{c_{3}\left(1+\tau d_{3} \exp \left(\tau d_{3}\right)\right)}_{c_{4}}+\left(b_{3}+h d_{3}\right) a_{n}^{0} \\
& +h \underbrace{\left(d_{3}+d_{3} b_{3} \exp \left(\tau d_{3}\right)+\tau d_{3}^{2} \exp \left(\tau d_{3}\right)\right)}_{d_{4}} \sum_{i=0}^{n-1} a_{i}^{0} \\
& \leq c_{4}+\underbrace{\left(b_{3}+\tau d_{3}\right)}_{b_{4}} a_{n}^{0}+h d_{4} \sum_{i=0}^{n-1} a_{i}^{0} .
\end{aligned}
$$

On the other hand, by integrating (13) from $t_{n}^{0}$ to $t \in \sigma_{n}^{0}$, we get,

$$
\begin{aligned}
a_{n}^{0} & \leq\left|u_{n-1}^{0}\left(t_{n}^{0}\right)\right|+h c_{1}+h A a_{n}^{0}+h b_{1}^{3} a_{n}^{0}+h^{2} d_{1} \sum_{i=0}^{n-1} \sum_{k=0}^{m} a_{i}^{k} \\
& \leq\left|u_{n-1}^{0}\left(t_{n}^{0}\right)\right|+h c_{1}+h b_{1} a_{n}^{0}+h^{2} d_{1} \sum_{i=0}^{n-1} a_{i}^{0}+h^{2} d_{1} \sum_{i=0}^{n-1} z_{i} .
\end{aligned}
$$

Moreover, from (3), we obtain,

$$
\begin{aligned}
\left|u_{n-1}^{0}\left(t_{n}^{0}\right)\right| & \leq a_{n-1}^{0}+h \sum_{j=1}^{m} a_{n-1}^{j} \\
& \leq a_{n-1}^{0}+h z_{n-1}
\end{aligned}
$$

hence,

$$
a_{n}^{0} \leq a_{n-1}^{0}+h z_{n-1}+h c_{1}+h b_{1} a_{n}^{0}+h^{2} d_{1} \sum_{i=0}^{n-1} a_{i}^{0}+h^{2} d_{1} \sum_{i=0}^{n-1} z_{i}
$$

using (24), we deduce that

$$
\begin{aligned}
a_{n}^{0} & \leq a_{n-1}^{0}+h\left(c_{4}+b_{4} a_{n-1}^{0}+h d_{4} \sum_{i=0}^{n-1} a_{i}^{0}\right)+h c_{1}+h b_{1} a_{n}^{0} \\
& +h^{2} d_{1} \sum_{i=0}^{n-1} a_{i}^{0}+h^{2} d_{1} \sum_{i=0}^{n-1}\left(c_{4}+b_{4} a_{i}^{0}+h d_{4} \sum_{k=0}^{n-1} a_{k}^{0}\right) \\
& \leq\left(1+h b_{4}\right) a_{n-1}^{0}+h \underbrace{\left(c_{4}+c_{1}+\tau d_{1} c_{4}\right)}_{c_{5}}+h b_{1} a_{n}^{0} \\
& +h^{2} \underbrace{\left(d_{4}+d_{1}+d_{1} b_{4}+\tau d_{1} d_{4}\right)}_{d_{5}} \sum_{i=0}^{n-1} a_{i}^{0},
\end{aligned}
$$


this implies that

$$
\left(1-h b_{1}\right) a_{n}^{0} \leq\left(1+h b_{4}\right) a_{n-1}^{0}+h c_{5}+h^{2} d_{5} \sum_{i=0}^{n-1} a_{i}^{0}
$$

hence, for all $h \in\left(0, \frac{1}{b_{1}}\right)$, we have

$$
a_{n}^{0} \leq \frac{1+h b_{4}}{1-h b_{1}} a_{n-1}^{0}+\frac{h^{2} d_{5}}{1-h b_{1}} \sum_{i=0}^{n-1} a_{i}^{0}+\frac{h c_{5}}{1-h b_{1}} .
$$

Then, by Lemma 3 , we get for all $n \in\{0,1, \ldots, N-1\}$

$$
\begin{aligned}
a_{n}^{0} & \leq \frac{a_{0}^{0}}{R_{2}-R_{1}}\left[\left(R_{2}-1\right) R_{2}^{n}+\left(1-R_{1}\right) R_{1}^{n}\right]+\frac{h c_{5}}{\left(R_{2}-R_{1}\right)\left(1-h b_{1}\right)}\left[R_{2}^{n}-R_{1}^{n}\right] \\
& \leq \frac{\alpha_{1}^{1}(m)}{R_{2}-R_{1}}\left[\left(R_{2}-1\right) R_{2}^{n}+\left(1-R_{1}\right) R_{1}^{n}\right]+\frac{h c_{5}}{\left(R_{2}-R_{1}\right)\left(1-h b_{1}\right)}\left[R_{2}^{n}-R_{1}^{n}\right]
\end{aligned}
$$

where

$$
\begin{aligned}
& R_{1}=\left(1+\frac{1+h b_{4}+h^{2} d_{5}}{1-h b_{1}}-h \sqrt{\frac{\left(b_{1}+b_{4}\right)^{2}+h^{2} d_{5}^{2}+2 d_{5}\left(1+h b_{4}\right)}{\left(1-h b_{1}\right)^{2}}+2 \frac{d_{5}}{1-h b_{1}}}\right) / 2, \\
& R_{2}=\left(1+\frac{1+h b_{4}+h^{2} d_{5}}{1-h b_{1}}+h \sqrt{\frac{\left(b_{1}+b_{4}\right)^{2}+h^{2} d_{5}^{2}+2 d_{5}\left(1+h b_{4}\right)}{\left(1-h b_{1}\right)^{2}}+2 \frac{d_{5}}{1-h b_{1}}}\right) / 2 .
\end{aligned}
$$

Since $0<R_{1} \leq 1 \leq R_{2}$, then for all $h \in\left(0, \frac{1}{b_{1}}\right)$, we have

$$
R_{1}^{n} \leq 1 \leq R_{2}^{n} \leq R_{2}^{N}=R_{2}^{\frac{\tau}{h}}, n=0,1, \ldots, N-1,
$$

which implies, from (25), that

$$
a_{n}^{0} \leq \alpha_{1}^{1}(m) \frac{\left(R_{2}-1\right) R_{2}^{\frac{\tau}{h}}+\left(1-R_{1}\right)}{R_{2}-R_{1}}+c_{5} \frac{h R_{2}^{\frac{\tau}{h}}}{\left(R_{2}-R_{1}\right)\left(1-h b_{1}\right)} .
$$

Then, there exist $h_{1} \in\left(0, \frac{1}{b_{1}}\right)$ and $\alpha_{1}^{2}(m) \geq 0$ such that for all $h \in\left(0, h_{1}\right]$

$$
a_{n}^{0} \leq \alpha_{1}^{2}(m), n=0,1, \ldots, N-1,
$$

which implies, from $(24)$, that for all $j=1,2, \ldots, m+1$ and $n=0,1, \ldots, N-1$

$$
a_{n}^{j} \leq z_{n} \leq c_{4}+b_{4} \alpha_{1}^{2}(m)+d_{4} \alpha_{1}^{2}(m) \tau=\alpha_{1}^{3}(m) .
$$

Hence, the first step is completed by setting

$$
\alpha_{1}(m)=\max \left(\alpha_{1}^{2}(m), \alpha_{1}^{3}(m)\right) .
$$

Claim 2. There exists a positive constant $\alpha(m)$ such that $\left\|\hat{u}_{n, p}^{(j)}\right\| \leq \alpha(m)$ for all $n=0,1, \ldots, N-1, j=0,1, \ldots, m+1$ and $p=1, \ldots, r-1$. 
Let $a_{n, p}^{j}=\left\|\hat{u}_{n, p}^{(j)}\right\|$ and $\xi_{p}=\max \left\{a_{i, p}^{j}, j=0, \ldots, m+1, i=0, \ldots, N-1\right\}$ for $p=$ $0, \ldots, r-1$.

Similarly to Claim 1 , by differentiating equation (15) $j$-times, we obtain for all $j=1, \ldots, m+1$,

$$
a_{0, p}^{j} \leq c_{1}+b_{1} \xi_{p-1}+d_{1} \sum_{l=0}^{j-1} a_{0, p}^{l}
$$

where $c_{1}, b_{1}, d_{1}$ are positive numbers.

On the other hand, by integrating (15) from $t_{0}^{p}$ to $t \in \sigma_{0}^{p}$, we get,

$$
a_{0, p}^{0} \leq c_{2}+b_{2} \xi_{p-1}+h d_{2} a_{0, p}^{0}
$$

where $c_{2}, b_{2}, d_{2}$ are positive numbers.

hence, there exists $h_{2} \in\left(0, h_{1}\right]$ and positive numbers $c_{3}, b_{3}, d_{3}$ such that for all $h \in\left(0, h_{2}\right]$ and $j \in\{0,1, \ldots, m+1\}$, we have

$$
a_{0, p}^{j} \leq c_{3}+b_{3} \xi_{p-1}+d_{3} \sum_{l=0}^{j-1} a_{0, p}^{l}
$$

Then, by Lemma 1 , for all $j \in\{0,1, \ldots, m+1\}$

$$
a_{0, p}^{j} \leq \underbrace{c_{3} \exp \left(d_{3}(m+1)\right)}_{c_{4}^{1}}+\underbrace{b_{3} \exp \left(d_{3}(m+1)\right)}_{b_{4}} \xi_{p-1},
$$

hence, for $c_{4}=\max \left(\alpha_{1}(m), c_{4}^{1}\right)$, we get for all $p=0,1, \ldots, r-1, j \in\{0,1, \ldots, m+1\}$

$$
a_{0, p}^{j} \leq c_{4}+b_{4} \xi_{p-1} .
$$

Next, by differentiating equation (17) $j$-times, we obtain for all $n=1, \ldots, N-1$ and $j=1, \ldots, m+1$,

$$
a_{n, p}^{j} \leq c_{5}+b_{5} \xi_{p-1}+e_{5} \sum_{l=0}^{j-1} a_{n, p}^{l}+d_{5} h \sum_{i=0}^{n-1} \sum_{l=0}^{m+1} a_{i, p}^{l}
$$

where $c_{5}, b_{5}, e_{5}, d_{5}$ are positive numbers.

Then, by Lemma 1 , for all $j \in\{1, \ldots, m+1\}$

$$
\begin{aligned}
a_{n, p}^{j} \leq \underbrace{c_{5} \exp \left(e_{5}(m+1)\right)}_{c_{6}} & +\underbrace{b_{5} \exp \left(e_{5}(m+1)\right)}_{b_{6}} \xi_{p-1}+\underbrace{e_{5} \exp \left(e_{5}(m+1)\right)}_{e_{6}} a_{n, p}^{0} \\
& +\underbrace{d_{5} \exp \left(e_{5}(m+1)\right)}_{d_{6}} h \sum_{i=0}^{n-1} \sum_{l=0}^{m+1} a_{i, p}^{l} .
\end{aligned}
$$


Consider the sequence $y_{n}=\sum_{j=1}^{m+1} a_{n, p}^{j}, n=0,1, \ldots, N-1$, hence, by the above inequality, the sequence $\left(y_{n}\right)$ satisfies for all $n=1, \ldots, N-1$,

$$
\begin{aligned}
y_{n} \leq \underbrace{(m+1) c_{6}}_{c_{7}^{1}} & +\underbrace{(m+1) b_{6}}_{b_{7}^{1}} \xi_{p-1}+\underbrace{(m+1) e_{6}}_{e_{7}} a_{n, p}^{0}+\underbrace{(m+1) d_{6}}_{d_{7}} h \sum_{i=0}^{n} a_{i, p}^{0} \\
& +\underbrace{(m+1) d_{6}}_{d_{7}} h \sum_{i=0}^{n-1} y_{i} .
\end{aligned}
$$

Moreover, from (26), we obtain,

$$
y_{0} \leq \underbrace{(m+1) c_{4}}_{c_{7}^{2}}+\underbrace{(m+1) b_{4}}_{b_{7}^{2}} \xi_{p-1}
$$

Let $c_{7}=\max \left\{c_{7}^{1}, c_{7}^{2}\right\}$ and $b_{7}=\max \left\{b_{7}^{1}, b_{7}^{2}\right\}$.

Then, from (27) and (28), we get for all $n=0,1, \ldots, N-1$,

$$
y_{n} \leq c_{7}+b_{7} \xi_{p-1}+e_{7} a_{n, p}^{0}+d_{7} h \sum_{i=0}^{n} a_{i, p}^{0}+d_{7} h \sum_{i=0}^{n-1} y_{i},
$$

hence, by Lemma 2, we obtain

$$
\begin{gathered}
y_{n} \leq \underbrace{c_{7}\left(1+\tau d_{7} \exp \left(\tau d_{7}\right)\right)}_{c_{8}}+\underbrace{b_{7}\left(1+\tau d_{7} \exp \left(\tau d_{7}\right)\right)}_{b_{8}} \xi_{p-1}+\underbrace{\left(e_{7}+\tau d_{7}\right)}_{e_{8}} a_{n, p}^{0} \\
+h \underbrace{\left(d_{7}+d_{7} e_{7} \exp \left(\tau d_{7}\right)+\tau d_{7}^{2} \exp \left(\tau d_{7}\right)\right)}_{d_{8}} \sum_{i=0}^{n-1} a_{i, p}^{0} .
\end{gathered}
$$

On the other hand, by integrating (17) from $t_{n}^{p}$ to $t \in \sigma_{n}^{p}$, we get

$$
\begin{aligned}
a_{n, p}^{0} & \leq\left|u_{n-1}^{p}\left(t_{n}^{p}\right)\right|+h c_{9}+h b_{9} \xi_{p-1}+h e_{9} a_{n, p}^{0}+d_{9} h^{2} \sum_{i=0}^{n-1} \sum_{l=0}^{m} a_{i, p}^{l} \\
& \leq\left|u_{n-1}^{p}\left(t_{n}^{p}\right)\right|+h c_{9}+h b_{9} \xi_{p-1}+h e_{9} a_{n, p}^{0}+d_{9} h^{2} \sum_{i=0}^{n-1} a_{i, p}^{0}+d_{9} h^{2} \sum_{i=0}^{n-1} y_{i}
\end{aligned}
$$

for all $n=1, \ldots, N-1$, where $c_{9}, b_{9}, e_{9}, d_{9}$ are positive numbers.

Moreover, from (9), we obtain,

$$
\begin{aligned}
\left|u_{n-1}^{p}\left(t_{n}^{p}\right)\right| & \leq a_{n-1, p}^{0}+h \sum_{j=1}^{m} a_{n-1, p}^{j} \\
& \leq a_{n-1, p}^{0}+h y_{n-1},
\end{aligned}
$$


then,

$$
a_{n, p}^{0} \leq a_{n-1, p}^{0}+h y_{n-1}+h c_{9}+h b_{9} \xi_{p-1}+h e_{9} a_{n, p}^{0}+d_{9} h^{2} \sum_{i=0}^{n-1} a_{i, p}^{0}+d_{9} h^{2} \sum_{i=0}^{n-1} y_{i},
$$

which implies, by using (29), that

$$
\begin{aligned}
\left(1-h e_{9}\right) a_{n, p}^{0} \leq & a_{n-1, p}^{0}+h\left(c_{8}+b_{8} \xi_{p-1}+e_{8} a_{n-1, p}^{0}+h d_{8} \sum_{i=0}^{n-1} a_{i, p}^{0}\right)+h c_{9}+h b_{9} \xi_{p-1} \\
& +d_{9} h^{2} \sum_{i=0}^{n-1} a_{i, p}^{0}+d_{9} h^{2} \sum_{i=0}^{n-1}\left(c_{8}+b_{8} \xi_{p-1}+e_{8} a_{i, p}^{0}+h d_{8} \sum_{k=0}^{n-1} a_{k, p}^{0}\right) \\
\leq & \left(1+h e_{8}\right) a_{n-1, p}^{0}+h \underbrace{\left(c_{8}+c_{9}+\tau d_{9} c_{8}\right)}_{c_{10}}+h \underbrace{\left(b_{8}+b_{9}+\tau d_{9} b_{8}\right)}_{b_{10}} \xi_{p-1} \\
& +h^{2} \underbrace{\left(d_{8}+d_{9}+d_{9} e_{8}+\tau d_{9} d_{8}\right)}_{d_{10}} \sum_{i=0}^{n-1} a_{i, p}^{0},
\end{aligned}
$$

hence, there exists $h_{3} \in\left(0, h_{2}\right]$ such that for all $h \in\left(0, h_{3}\right]$, we have

$$
a_{n, p}^{0} \leq \frac{1+h e_{8}}{1-h e_{9}} a_{n-1, p}^{0}+\frac{h\left(c_{10}+b_{10} \xi_{p-1}\right)}{1-h e_{9}}+\frac{h^{2} d_{10}}{1-h e_{9}} \sum_{i=0}^{n-1} a_{i, p}^{0} .
$$

Then, by Lemma 3, we get for all $n \in\{0,1, \ldots, N-1\}$

$$
a_{n, p}^{0} \leq \frac{a_{0, p}^{0}}{R_{2}-R_{1}}\left[\left(R_{2}-1\right) R_{2}^{n}+\left(1-R_{1}\right) R_{1}^{n}\right]+\frac{h\left(c_{10}+b_{10} \xi_{p-1}\right)}{\left(R_{2}-R_{1}\right)\left(1-h e_{9}\right)}\left[R_{2}^{n}-R_{1}^{n}\right],
$$

where

$R_{1}=\left(1+\frac{1+h e_{8}+h^{2} d_{10}}{1-h e_{9}}-h \sqrt{\frac{\left(e_{8}+e_{9}\right)^{2}+h^{2} d_{10}^{2}+2 d_{10}\left(1+h e_{8}\right)}{\left(1-h e_{9}\right)^{2}}+2 \frac{d_{10}}{1-h b_{9}}}\right) / 2$,
$R_{2}=\left(1+\frac{1+h e_{8}+h^{2} d_{10}}{1-h e_{9}}+h \sqrt{\frac{\left(e_{8}+e_{9}\right)^{2}+h^{2} d_{10}^{2}+2 d_{10}\left(1+h e_{8}\right)}{\left(1-h e_{9}\right)^{2}}+2 \frac{d_{10}}{1-h b_{9}}}\right) / 2$.

Hence, similar as in $(25)$, there exist $h_{4} \in\left(0, h_{3}\right]$ and $\bar{R}>0$ such that for all $h \in\left(0, h_{4}\right]$, we have

$$
a_{n, p}^{0} \leq a_{0, p}^{0} \bar{R}+\left(c_{10}+b_{10} \xi_{p-1}\right) \bar{R}
$$

which implies, by $\operatorname{using}(26)$, that for all $n \in\{0,1, \ldots, N-1\}$ and $p \in\{0,1, \ldots, r-$ $1\}$

$$
a_{n, p}^{0} \leq \underbrace{\left(c_{4}+c_{10}\right) \bar{R}}_{c_{11}}+\underbrace{\left(b_{4}+b_{10}\right) \bar{R}}_{b_{11}} \xi_{p-1} .
$$


Then, from (29), we get for all $n \in\{0,1, \ldots, N-1\}, j \in\{1, \ldots, m+1\}$ and $p \in$ $\{0,1, \ldots, r-1\}$,

$$
a_{n, p}^{j} \leq y_{n} \leq \underbrace{\left(c_{8}+e_{8} c_{11}+\tau d_{8} c_{11}\right)}_{c_{12}^{1}}+\underbrace{\left(b_{8}+e_{8} b_{11}+\tau d_{8} b_{11}\right)}_{b_{12}^{1}} \xi_{p-1},
$$

Let $c_{12}=\max \left(c_{11}, c_{12}^{1}\right)$ and $b_{12}=\max \left(b_{11}, b_{12}^{1}\right)$.

We deduce that, for all $p \in\{0,1, \ldots, r-1\}$,

$$
\begin{aligned}
\xi_{p} & \leq c_{12}+b_{12} \xi_{p-1} \\
& \leq c_{12}+b_{12} \sum_{i=0}^{p-1} \xi_{i} .
\end{aligned}
$$

Then, by Lemma 1 , we get for all $p \in\{0,1, \ldots, r-1\}, n \in\{0,1, \ldots, N-1\}$ and $j \in\{0,1, \ldots, m+1\}$,

$$
a_{n, p}^{j} \leq \xi_{p} \leq c_{12} \exp \left(r b_{12}\right)=\alpha(m)
$$

This completes the proof of Lemma 4.

The following theorem describes the order of convergence of the method.

Theorem 1 Let $g, k, a, b$ and $\Phi$ be $m$ times continuously differentiable on their respective domains. Then equations $(2),(3),(6),(9)$ define a unique approximation $u \in S_{m}^{(0)}\left(\Pi_{N}\right)$, and the resulting error function $e:=y-u$ satisfies:

$$
\|e\| \leq C h^{m}
$$

provided that $h$ is sufficiently small, where $C$ is a finite constant independent of $h$.

Proof. The proof is split into two steps.

Claim 1. There exists a constant $C_{0}$ independent of $h$ such that $\left\|e^{0}\right\| \leq C_{0} h^{m}$, where the error $e^{0}=\left.e\right|_{\sigma^{0}}$ which is defined on $\sigma_{n}^{0}$, by $e^{0}(t)=e_{n}^{0}(t)=y(t)-u_{n}^{0}(t)$ for all $n \in\{0,1, \ldots, N-1\}$.

Let $t \in \sigma_{0}^{0}$, we have from Lemma 4 , for sufficient small $h$

$$
\left\|e_{0}^{0}(t)\right\|=\left\|y(t)-u_{0}^{0}(t)\right\| \leq \frac{\left\|y^{(m+1)}\right\|}{(m+1) !} h^{m+1} \leq \frac{\alpha(m)}{(m+1) !} h^{m+1} .
$$

In general for $n=1,2, \ldots, N-1$ and $t \in \sigma_{n}^{0}$, we have from (13),

$$
\begin{aligned}
y^{\prime}(t)-\hat{u}_{n, 0}^{\prime}(t)=a(t)\left(y(t)-\hat{u}_{n, 0}(t)\right) & +\sum_{i=0}^{n-1} \int_{t_{i}^{0}}^{t_{i+1}^{0}} k(t, s)\left(y(s)-u_{i}^{0}(s)\right) d s \\
& +\int_{t_{n}^{0}}^{t} k(t, s)\left(y(s)-\hat{u}_{n, 0}(s)\right) d s
\end{aligned}
$$


this implies that,

$$
\left\|y^{\prime}-\hat{u}_{n, 0}^{\prime}\right\| \leq h k_{0} \sum_{i=0}^{n-1}\left\|e_{i}^{0}\right\|+\underbrace{\left(A+\tau k_{0}\right)}_{\widetilde{A}}\left\|y-\hat{u}_{n, 0}\right\|,
$$

where, $k_{0}=\|k\|_{L^{\infty}}\left(\sigma^{0} \times \sigma^{0}\right)$.

On the other hand, for $t \in \sigma_{n}^{0}$,

$$
\begin{aligned}
y(t)-\hat{u}_{n, 0}(t) & =y\left(t_{n}^{0}\right)-\hat{u}_{n, 0}\left(t_{n}^{0}\right)+\int_{t_{n}^{0}}^{t}\left(y^{\prime}(s)-\hat{u}_{n, 0}^{\prime}(s)\right) d s \\
& =e_{n-1}^{0}\left(t_{n}^{0}\right)+\int_{t_{n}^{0}}^{t}\left(y^{\prime}(s)-\hat{u}_{n, 0}^{\prime}(s)\right) d s,
\end{aligned}
$$

it follows that,

$$
\left\|y-\hat{u}_{n, 0}\right\| \leq\left\|e_{n-1}^{0}\right\|+h\left\|y^{\prime}-\hat{u}_{n, 0}^{\prime}\right\|,
$$

hence, by using (30), we get,

$$
\left\|y-\hat{u}_{n, 0}\right\| \leq \frac{1}{1-h \widetilde{A}}\left\|e_{n-1}^{0}\right\|+\frac{h^{2} k_{0}}{1-h \widetilde{A}} \sum_{i=0}^{n-1}\left\|e_{i}^{0}\right\|,
$$

Therefore, by Lemma 4,

$$
\begin{aligned}
\left\|e_{n}^{0}\right\| & \leq\left\|y-\widehat{u}_{n, 0}\right\|+\left\|\widehat{u}_{n, 0}-u_{n}^{0}\right\| \\
& \leq\left\|y-\widehat{u}_{n, 0}\right\|+\frac{\alpha(m)}{(m+1) !} h^{m+1} .
\end{aligned}
$$

Then,

$$
\left\|e_{n}^{0}\right\| \leq \frac{1}{1-h \widetilde{A}}\left\|e_{n-1}^{0}\right\|+\frac{h^{2} k_{0}}{1-h \widetilde{A}} \sum_{i=0}^{n-1}\left\|e_{i}^{0}\right\|+\frac{\alpha(m)}{(m+1) !} h^{m+1},
$$

hence by Lemma 3 , for all $n \in\{0,1, \ldots, N-1\}$

$$
\begin{aligned}
\left\|e_{n}^{0}\right\| & \leq \frac{\left\|e_{0}^{0}\right\|}{R_{2}-R_{1}}\left[\left(R_{2}-1\right) R_{2}^{n}+\left(1-R_{1}\right) R_{1}^{n}\right]+\frac{\frac{\alpha(m)}{(m+1) !} h^{m+1}}{R_{2}-R_{1}}\left[R_{2}^{n}-R_{1}^{n}\right] \\
& \leq \frac{\alpha(m)}{(m+1) !} h^{m+1} \frac{\left[\left(R_{2}-1\right) R_{2}^{n}+\left(1-R_{1}\right) R_{1}^{n}\right]+\left[R_{2}^{n}-R_{1}^{n}\right]}{R_{2}-R_{1}}
\end{aligned}
$$

where

$$
\begin{aligned}
R_{1} & =\left(1+\frac{1+h^{2} k_{0}}{1-h \widetilde{A}}-\frac{h}{1-h \widetilde{A}} \sqrt{\xi}\right) / 2=\frac{1-h(\widetilde{A}+\sqrt{\xi})}{2(1-h \widetilde{A})} \\
R_{2} & =\left(1+\frac{1+h^{2} k_{0}}{1-h \widetilde{A}}+\frac{h}{1-h \widetilde{A}} \sqrt{\xi}\right) / 2=\frac{1-h(\widetilde{A}-\sqrt{\xi})}{2(1-h \widetilde{A})} \\
\xi & =\widetilde{A}^{2}+\left(h k_{0}\right)^{2}+2 k_{0}(2-h \widetilde{A}) .
\end{aligned}
$$


Since $0<R_{1} \leq 1 \leq R_{2}$, then

$$
R_{1}^{n} \leq 1 \leq R_{2}^{n} \leq R_{2}^{N}=R_{2}^{\frac{\tau}{h}}, n=0,1, \ldots, N-1,
$$

which implies, from (31), that

$$
\left\|e_{n}^{0}\right\| \leq \frac{\alpha(m)}{(m+1) !} h^{m}(1-h \widetilde{A}) \frac{\left[\left(R_{2}-1\right) R_{2}^{\frac{\tau}{h}}+\left(1-R_{1}\right)\right]+R_{2}^{\frac{\tau}{h}}}{\sqrt{\xi}},
$$

we deduce that, there exist $\widetilde{R}_{1}$ and $h_{1}$ such that, for all $h \in\left(0, h_{1}\right]$

$$
\left\|e_{n}^{0}\right\| \leq \widetilde{R}_{1} h^{m}
$$

Thus,

$$
\left\|e^{0}\right\|=\max _{n=0, \ldots, N-1}\left\|e_{n}^{0}\right\| \leq \widetilde{R}_{1} h^{m}
$$

Hence, the first step is completed by taking $C_{0}=\widetilde{R}_{1}$.

Claim 2. There exists a constant $C$ independent of $h$ such that $\|e\| \leq C h^{m}$. Define the error $e^{p}(t)$ on $\sigma^{p}$ by $e^{p}(t)=y(t)-u^{p}(t)$ and on $\sigma_{n}^{p}$ by $e^{p}(t)=e_{n}^{p}(t)=$ $y(t)-u_{n}^{p}(t)$ for all $n \in\{0,1, \ldots, N-1\}$ and $p \in\{0,1, \ldots, r-1\}$.

First, let $t \in \sigma_{0}^{p}$, we have from (15),

$$
\begin{aligned}
y^{\prime}(t)-\hat{u}_{0, p}^{\prime}(t) & =a(t)\left(y(t)-\hat{u}_{0, p}(t)\right)+b(t)\left(e_{0}^{p-1}(t-\tau)\right) \\
& +\int_{t-\tau}^{t_{0}^{p}} k(t, s) e^{p-1}(s) d s+\int_{t_{0}^{p}}^{t} k(t, s)\left(y(s)-\hat{u}_{0, p}(s)\right) d s,
\end{aligned}
$$

such that $y\left(t_{0}^{p}\right)-\hat{u}_{0, p}\left(t_{0}^{p}\right)=y\left(t_{0}^{p}\right)-u^{p-1}\left(t_{0}^{p}\right)=e^{p-1}\left(t_{0}^{p}\right)$,

hence,

$$
\begin{aligned}
\left\|y-\hat{u}_{0, p}\right\| & \leq\left\|e^{p-1}\right\|+h\left\|y^{\prime}-\hat{u}_{0, p}^{\prime}\right\| \\
& \leq\left\|e^{p-1}\right\|+h(\underbrace{\left(B+\tau k_{0}\right)}_{\widetilde{B}}\left\|e^{p-1}\right\|+\widetilde{A}\left\|y-\hat{u}_{0, p}\right\|),
\end{aligned}
$$

where $B=\|b\|$ and $\widetilde{A}=A+\tau k_{0}$, this implies that

$$
\left\|y-\hat{u}_{0, p}\right\| \leq \frac{1+h \widetilde{B}}{1-h \widetilde{A}}\left\|e^{p-1}\right\|
$$

Therefore, by Lemma 4,

$$
\begin{aligned}
\left\|e_{0}^{p}\right\| & \leq\left\|y-\widehat{u}_{0, p}\right\|+\left\|\widehat{u}_{0, p}-u_{0}^{p}\right\| \\
& \leq\left\|y-\widehat{u}_{0, p}\right\|+\frac{\alpha(m)}{(m+1) !} h^{m+1}
\end{aligned}
$$


then,

$$
\left\|e_{0}^{p}\right\| \leq \frac{1+h \widetilde{B}}{1-h \widetilde{A}}\left\|e^{p-1}\right\|+\frac{\alpha(m)}{(m+1) !} h^{m+1} .
$$

Next, let $t \in \sigma_{n}^{p}$ for $n \in\{1,2, \ldots, n\}$, we have from (17)

$$
\begin{aligned}
y^{\prime}(t)-\hat{u}_{n, p}^{\prime}(t)=a(t)(y(t) & \left.-\hat{u}_{n, p}(t)\right)+b(t)\left(e_{n}^{p-1}(t-\tau)\right)+\int_{t-\tau}^{t_{0}^{p}} k(t, s) e^{p-1}(s) d s \\
& +\sum_{i=0}^{n-1} \int_{t_{i}^{p}}^{t_{i+1}^{p}} k(t, s) e_{i}^{p}(s) d s+\int_{t_{n}^{p}}^{t} k(t, s)\left(y(s)-\hat{u}_{n, p}(s)\right) d s,
\end{aligned}
$$

such that $y\left(t_{n}^{p}\right)-\hat{u}_{n, p}\left(t_{n}^{p}\right)=y\left(t_{n}^{p}\right)-u_{n-1}^{p}\left(t_{n}^{p}\right)=e_{n-1}^{p}\left(t_{n}^{p}\right)$,

hence,

$$
\begin{aligned}
\left\|y-\hat{u}_{n, p}\right\| & \leq\left\|e_{n-1}^{p}\right\|+h\left\|y^{\prime}-\hat{u}_{n, p}^{\prime}\right\| \\
& \leq\left\|e_{n-1}^{p}\right\|+h \widetilde{B}\left\|e^{p-1}\right\|+\widetilde{A}\left\|y-\hat{u}_{n, p}\right\|+h^{2} k_{0} \sum_{i=0}^{n-1}\left\|e_{i}^{p}\right\|,
\end{aligned}
$$

this implies that

$$
\left\|y-\hat{u}_{n, p}\right\| \leq \frac{1}{1-h \widetilde{A}}\left\|e_{n-1}^{p}\right\|+\frac{h \widetilde{B}}{1-h \widetilde{A}}\left\|e^{p-1}\right\|+\frac{h^{2} k_{0}}{1-h \widetilde{A}} \sum_{i=0}^{n-1}\left\|e_{i}^{p}\right\| .
$$

Then, by Lemma 4,

$$
\begin{aligned}
\left\|e_{n}^{p}\right\| & \leq\left\|y-\widehat{u}_{n, p}\right\|+\left\|\widehat{u}_{n, p}-u_{n}^{p}\right\| \\
& \leq \frac{1}{1-h \widetilde{A}}\left\|e_{n-1}^{p}\right\|+\frac{h \widetilde{B}}{1-h \widetilde{A}}\left\|e^{p-1}\right\|+\frac{h^{2} k_{0}}{1-h \widetilde{A}} \sum_{i=0}^{n-1}\left\|e_{i}^{p}\right\|+\frac{\alpha(m)}{(m+1) !} h^{m+1} .
\end{aligned}
$$

It follows from Lemma 3 , for all $n \in\{0,1, \ldots, N-1\}$,

$$
\begin{aligned}
\left\|e_{n}^{p}\right\| & \leq \frac{\left\|e_{0}^{p}\right\|}{R_{2}-R_{1}}\left[\left(R_{2}-1\right) R_{2}^{n}+\left(1-R_{1}\right) R_{1}^{n}\right]+\frac{\frac{h \widetilde{B}}{1-h \widetilde{A}}\left\|e^{p-1}\right\|+\frac{\alpha(m)}{(m+1) !} h^{m+1}}{R_{2}-R_{1}}\left[R_{2}^{n}-R_{1}^{n}\right] \\
& \leq\left\|e_{0}^{p}\right\| \frac{\left(R_{2}-1\right) R_{2}^{\tau}+\left(1-R_{1}\right)}{R_{2}-R_{1}}+\frac{\widetilde{B}\left\|e^{p-1}\right\|+(1-h \widetilde{A}) \frac{\alpha(m)}{(m+1) !} h^{m}}{\sqrt{\xi}} R_{2}^{\frac{\tau}{h}},
\end{aligned}
$$

where $R_{1}$ and $R_{2}$ are defined by (32).

So, there exist $\widetilde{R}_{2}$ and $h_{2}$ such that, for all $h \in\left(0, h_{2}\right]$

$$
\left\|e_{n}^{p}\right\| \leq\left(\left\|e_{0}^{p}\right\|+\left\|e^{p-1}\right\|+h^{m}\right) \widetilde{R}_{2},
$$

which implies, by (34), that for all $h \leq h_{2}$,

$$
\begin{aligned}
\left\|e_{n}^{p}\right\| & \leq\left(\left(\frac{1+h \widetilde{B}}{1-h \widetilde{A}}+1\right)\left\|e^{p-1}\right\|+\frac{\alpha(m)}{(m+1) !} h^{m+1}+h^{m}\right) \widetilde{R}_{2} \\
& \leq\left(\left(\frac{1+h_{2} \widetilde{B}}{1-h_{2} \widetilde{A}}+1\right)\left\|e^{p-1}\right\|+\left(\frac{\alpha(m)}{(m+1) !} h_{2}+1\right) h^{m}\right) \widetilde{R}_{2},
\end{aligned}
$$


hence, for $\widetilde{R}_{3}=\max \left\{\left(\frac{1+h_{2} \widetilde{B}}{1-h_{2} \widetilde{A}}+1\right) \widetilde{R}_{2},\left(\frac{\alpha(m)}{(m+1) !} h_{2}+1\right) \widetilde{R}_{2}\right\}$, we obtain,

$$
\left\|e_{n}^{p}\right\| \leq \widetilde{R}_{3}\left\|e^{p-1}\right\|+\widetilde{R}_{3} h^{m}
$$

we deduce that,

$$
\left\|e^{p}\right\| \leq \widetilde{R}_{3} \sum_{i=0}^{p-1}\left\|e^{i}\right\|+\widetilde{R}_{3} h^{m} \leq \widetilde{R}_{3} \sum_{i=0}^{p-1}\left\|e^{i}\right\|+\widetilde{R}_{4} h^{m}
$$

where $\widetilde{R}_{4}=\max \left\{\widetilde{R}_{1}, \widetilde{R}_{3}\right\}$.

Then, from (33), (35) and by using Lemma 1, we get,

$$
\left\|e^{p}\right\| \leq \widetilde{R}_{4} h^{m} \exp \left(r \widetilde{R}_{3}\right) .
$$

Thus, the proof is completed by taking $C=\widetilde{R}_{4} \exp \left(r \widetilde{R}_{3}\right)$.

\section{Numerical Examples}

In this section, we present several examples with analytical solutions to show the performance of the described method in Section 2 for solving the system of Eqs. (1). In each example, we present a different dimensional nonlinear system (one-dimensional in example (1), two-dimensional in examples (2), (3) , (4), (5), (6), four-dimensional in example (8) and five-dimensional in examples (7) and (9)). We calculate the error between $y$ and $u$. Also, the results obtained in examples (3), (4), (5), and (6), which arise in mathematical modeling of "predator-prey" dynamics in Ecology, are compared with those obtained from the application of Variational Iteration Method (VIM) [20], Adomian Decomposition Method (ADM) [3] and Pseudospectral Legendre Method (PLM) [25]. Moreover, we apply two mathematical models that simulate the evolution of the COVID-19 epidemic in China, namely the SEIR [18] model in example (8) and the SEIRU [22] model in example (9).

Example 1 ([23]) Consider the Volterra delay integro-differential equation $y^{\prime}(t)=-(6+\sin (t)) y(t)+y\left(t-\frac{\pi}{4}\right)-\int_{t-\frac{\pi}{4}}^{t} \sin (s) y(s) d s$, for $t \in\left[0, \frac{\pi}{2}\right]$ and $y(t)=e^{\cos (t)}$ for $t \in\left[-\frac{\pi}{4}, 0\right]$ with $\Phi(t)=e^{\cos (t)}$. The absolute errors for $(N, m)=\{(4,4),(6,6),(8,8),(10,10)\}$ are presented in Table 1.

Example 2 ([2]) Consider the nonlinear system of VDIDEs:

$$
\left(\begin{array}{l}
y_{1}^{\prime}(t) \\
y_{2}^{\prime}(t)
\end{array}\right)=-4\left(\begin{array}{l}
y_{1}(t) \\
y_{2}(t)
\end{array}\right)+\left(\begin{array}{cc}
0 & \sin (t) \\
\cos (t) & 0
\end{array}\right)\left(\begin{array}{l}
y_{1}\left(t-\frac{\pi}{5}\right) \\
y_{2}\left(t-\frac{\pi}{5}\right)
\end{array}\right)+\int_{t-\frac{\pi}{5}}^{t}\left(\begin{array}{l}
\frac{\left(1+\sin ^{2}(s) y_{1}^{2}(s)\right)}{\sqrt{2}\left(1+y_{1}^{2}(s)\right)} \\
\frac{\left(1+\cos ^{2}(s) y_{2}^{2}(s)\right)}{\sqrt{2}\left(1+y_{2}^{2}(s)\right.}
\end{array}\right) d s+\left(\begin{array}{l}
g_{1}(t) \\
g_{2}(t)
\end{array}\right)
$$

for $t \in\left[0, \frac{2 \pi}{5}\right], g(t)=\left(g_{1}(t), g_{2}(t)\right)^{t}$ is chosen so that the exact solution is $y(t)=(\sin (t), \cos (t))$ and $\Phi(t)=y(t)$ for $t \in\left[-\frac{\pi}{5}, 0\right]$

The absolute errors for $(N, m)=\{(4,4),(8,8)\}$ are presented in Table 2 . 
Table 1: Absolute errors for $y(t)$ of Example 1

\begin{tabular}{lllll}
\hline$t$ & $(N, m)=(4,4)$ & $(N, m)=(6,6)$ & $(N, m)=(8,8)$ & $(N, m)=(10,10)$ \\
\hline 0.0 & 0.0 & 0.0 & 0.0 & 0.0 \\
$\pi / 10$ & $1.79 \times 10^{-6}$ & $2.58 \times 10^{-10}$ & $2.76 \times 10^{-10}$ & $7.68 \times 10^{-11}$ \\
$\pi / 9$ & $3.68 \times 10^{-5}$ & $3.21 \times 10^{-8}$ & $3.02 \times 10^{-10}$ & $1.33 \times 10^{-10}$ \\
$\pi / 8$ & $6.54 \times 10^{-5}$ & $1.16 \times 10^{-7}$ & $3.76 \times 10^{-10}$ & $1.99 \times 10^{-10}$ \\
$\pi / 7$ & $3.60 \times 10^{-5}$ & $6.21 \times 10^{-8}$ & $6.41 \times 10^{-10}$ & $4.02 \times 10^{-10}$ \\
$\pi / 6$ & $1.22 \times 10^{-5}$ & $1.61 \times 10^{-8}$ & $2.39 \times 10^{-10}$ & $3.35 \times 10^{-11}$ \\
$\pi / 5$ & $3.95 \times 10^{-5}$ & $2.17 \times 10^{-8}$ & $1.89 \times 10^{-9}$ & $2.50 \times 10^{-10}$ \\
$\pi / 4$ & $2.12 \times 10^{-4}$ & $1.26 \times 10^{-6}$ & $3.40 \times 10^{-8}$ & $9.93 \times 10^{-10}$ \\
$\pi / 3$ & $2.16 \times 10^{-4}$ & $5.35 \times 10^{-6}$ & $1.96 \times 10^{-8}$ & $1.52 \times 10^{-7}$ \\
$\pi / 2$ & $1.59 \times 10^{-2}$ & $2.44 \times 10^{-4}$ & $3.00 \times 10^{-6}$ & $4.31 \times 10^{-7}$
\end{tabular}

Table 2: Absolute errors for $y(t)$ of Example 2

\begin{tabular}{lll|ll}
\hline$t$ & \multicolumn{2}{c|}{$y_{1}(t)$} & \multicolumn{2}{c}{$y_{2}(t)$} \\
& $(N, m)=(4,4)$ & $(N, m)=(8,8)$ & $(N, m)=(4,4)$ & $(N, m)=(8,8)$ \\
\hline 0.0 & 0.0 & 0.0 & 0.0 & 0.0 \\
$\pi / 20$ & $7.96 \times 10^{-7}$ & $1.56 \times 10^{-14}$ & $2.08 \times 10^{-8}$ & $1.22 \times 10^{-12}$ \\
$2 \pi / 20$ & $3.07 \times 10^{-6}$ & $1.48 \times 10^{-11}$ & $1.62 \times 10^{-8}$ & $1.66 \times 10^{-10}$ \\
$3 \pi / 20$ & $3.46 \times 10^{-6}$ & $2.84 \times 10^{-11}$ & $2.04 \times 10^{-7}$ & $2.88 \times 10^{-10}$ \\
$4 \pi / 20$ & $6.39 \times 10^{-6}$ & $1.72 \times 10^{-11}$ & $1.63 \times 10^{-7}$ & $3.55 \times 10^{-10}$ \\
$5 \pi / 20$ & $7.17 \times 10^{-6}$ & $1.40 \times 10^{-10}$ & $8.12 \times 10^{-7}$ & $2.42 \times 10^{-10}$ \\
$6 \pi / 20$ & $1.16 \times 10^{-5}$ & $3.07 \times 10^{-12}$ & $5.98 \times 10^{-7}$ & $1.79 \times 10^{-10}$ \\
$7 \pi / 20$ & $1.32 \times 10^{-5}$ & $5.08 \times 10^{-11}$ & $1.38 \times 10^{-6}$ & $3.17 \times 10^{-11}$ \\
$8 \pi / 20$ & $2.01 \times 10^{-5}$ & $1.49 \times 10^{-10}$ & $4.73 \times 10^{-7}$ & $2.37 \times 10^{-10}$ \\
\hline
\end{tabular}

Example 3 ([25]) In this example, we solve the system (1) of two nonlinear delay integro-differential equations with $\Phi_{1}(t)=-t^{2}, \Phi_{2}(t)=\frac{1}{2} t e^{-t}$ for $t \in\left[-\frac{1}{3}, 0\right]$, $k_{1}(t, s, y(t), y(s))=(3-2(t-s)) y_{1}(t) y_{2}(s)$, $k_{2}(t, s, y(t), y(s))=(t-s) y_{1}(s) y_{2}(t)$,

$f_{1}(t, y(t))=t^{2}\left(2-3 t e^{-t}-\frac{7}{2} e^{-t}+\frac{13}{6} t e^{\frac{1}{3}-t}+\frac{22}{9} e^{\frac{1}{3}-t}\right)-2 t+y_{1}(t)\left(2-y_{2}(t)\right)$, $f_{2}(t, y(t))=\frac{1}{648} e^{-t}\left(342 t^{3}-8 t^{2}+325 t+324\right)+y_{2}(t)\left(-2+y_{1}(t)\right)$.

The exact solution of this system is in the following form $y_{1}(t)=-t^{2}, y_{2}(t)=$ $\frac{1}{2} t e^{-t}$.

The absolute errors of Taylor Collocation Method (TCM) for $(N, m)=(8,8)$ are compared with the absolute error of the Variational Iteration Method (VIM), Adomian Decomposition Method (ADM) and Pseudospectral Legendre Method (PLM) given in [25] in Table 3 and Table 4.

Example 4 ([25]) Consider the system (1) of two nonlinear delay integrodifferential equations

$$
\begin{aligned}
& y_{1}^{\prime}(t)=\left(-\frac{5}{2} t^{3}+\frac{49}{12} t^{2}+\frac{17}{12} t-\frac{23}{6}\right)+y_{1}(t)\left(1-\frac{1}{3} y_{2}(t)\right)+\int_{t-\frac{1}{2}}^{t}-y_{1}(t) y_{2}(s) d s \\
& y_{2}^{\prime}(t)=\left(\frac{15}{8} t^{3}-\frac{1}{4} t^{2}+\frac{3}{8} t-1\right)+y_{2}(t)\left(-2+y_{1}(t)\right)+\int_{t-\frac{1}{2}}^{t}(t-s-1) y_{1}(s) y_{2}(t) d s, \\
& \text { for } t \in[0,1] \text { and } y(t)=\Phi(t) \text { for } t \in\left[-\frac{1}{2}, 0\right] \text { with } \\
& \Phi_{1}(t)=-3 t+1, \Phi_{2}(t)=t^{2}-t,
\end{aligned}
$$


Table 3: Comparison of the absolute errors for $y_{1}(t)$ of Example 3

\begin{tabular}{lllll}
\hline $\mathrm{t}$ & PLM & ADM & VIM & TCM \\
\hline 0.1 & $1.02 \times 10^{-4}$ & $1.68 \times 10^{-6}$ & $4.50 \times 10^{-10}$ & $1.40 \times 10^{-13}$ \\
0.2 & $1.76 \times 10^{-4}$ & $2.56 \times 10^{-6}$ & $4.07 \times 10^{-9}$ & $2.75 \times 10^{-12}$ \\
0.3 & $2.29 \times 10^{-4}$ & $3.70 \times 10^{-5}$ & $4.72 \times 10^{-8}$ & $1.21 \times 10^{-11}$ \\
0.4 & $2.69 \times 10^{-4}$ & $1.88 \times 10^{-4}$ & $3.64 \times 10^{-7}$ & $6.08 \times 10^{-12}$ \\
0.5 & $3.04 \times 10^{-4}$ & $6.92 \times 10^{-4}$ & $2.03 \times 10^{-6}$ & $6.66 \times 10^{-15}$ \\
0.6 & $3.42 \times 10^{-4}$ & $2.07 \times 10^{-3}$ & $8.80 \times 10^{-6}$ & $3.39 \times 10^{-11}$ \\
0.7 & $3.90 \times 10^{-4}$ & $5.27 \times 10^{-3}$ & $3.12 \times 10^{-5}$ & $5.61 \times 10^{-12}$ \\
0.8 & $4.56 \times 10^{-4}$ & $1.17 \times 10^{-2}$ & $9.44 \times 10^{-5}$ & $1.02 \times 10^{-10}$ \\
0.9 & $5.48 \times 10^{-4}$ & $2.39 \times 10^{-2}$ & $2.51 \times 10^{-4}$ & $9.29 \times 10^{-11}$ \\
1.0 & $6.74 \times 10^{-4}$ & $4.51 \times 10^{-2}$ & $6.04 \times 10^{-4}$ & $1.49 \times 10^{-10}$ \\
\hline
\end{tabular}

Table 4: Comparison of the absolute errors for $y_{2}(t)$ of Example 3

\begin{tabular}{lllll}
\hline $\mathrm{t}$ & PLM & ADM & VIM & Present method \\
\hline 0.1 & $1.76 \times 10^{-3}$ & $2.53 \times 10^{-6}$ & $9.80 \times 10^{-8}$ & $4.82 \times 10^{-15}$ \\
0.2 & $2.20 \times 10^{-3}$ & $2.12 \times 10^{-5}$ & $6.93 \times 10^{-8}$ & $2.88 \times 10^{-13}$ \\
0.3 & $1.91 \times 10^{-3}$ & $1.50 \times 10^{-4}$ & $2.69 \times 10^{-7}$ & $1.43 \times 10^{-12}$ \\
0.4 & $1.33 \times 10^{-3}$ & $6.29 \times 10^{-4}$ & $3.55 \times 10^{-7}$ & $2.82 \times 10^{-12}$ \\
0.5 & $7.39 \times 10^{-4}$ & $1.89 \times 10^{-3}$ & $2.49 \times 10^{-6}$ & $9.11 \times 10^{-12}$ \\
0.6 & $3.17 \times 10^{-4}$ & $4.69 \times 10^{-3}$ & $1.08 \times 10^{-5}$ & $1.13 \times 10^{-11}$ \\
0.7 & $1.30 \times 10^{-4}$ & $1.00 \times 10^{-2}$ & $3.85 \times 10^{-5}$ & $9.67 \times 10^{-12}$ \\
0.8 & $1.51 \times 10^{-4}$ & $1.95 \times 10^{-2}$ & $1.14 \times 10^{-4}$ & $1.64 \times 10^{-11}$ \\
0.9 & $2.70 \times 10^{-4}$ & $3.48 \times 10^{-2}$ & $3.00 \times 10^{-4}$ & $2.63 \times 10^{-11}$ \\
1.0 & $3.08 \times 10^{-4}$ & $5.84 \times 10^{-2}$ & $7.11 \times 10^{-4}$ & $3.76 \times 10^{-11}$ \\
\hline
\end{tabular}

$k_{1}(t, s, y(t), y(s))=-y_{1}(t) y_{2}(s)$,

$k_{2}(t, s, y(t), y(s))=(t-s-1) y_{1}(s) y_{2}(t)$,

$f_{1}(t, y(t))=-\frac{5}{2} t^{3}+\frac{49}{12} t^{2}+\frac{17}{12} t-\frac{23}{6}+y_{1}(t)\left(1-\frac{1}{3} y_{2}(t)\right)$,

$f_{2}(t, y(t))=\frac{15}{8} t^{3}-\frac{1}{4} t^{2}+\frac{3}{8} t-1+y_{2}(t)\left(-2+y_{1}(t)\right)$.

The exact solution of this system is in the following form

$y_{1}(t)=-3 t+1, y_{2}(t)=t^{2}-t$.

The absolute errors of Taylor Collocation Method (TCM) for $m=10, N=10$ are compared with the absolute error of the Variational Iteration Method (VIM), Adomian Decomposition Method (ADM) and Pseudospectral Legendre Method (PLM) given in [25] in Table 5 and Table 6.

Example 5 ([25]) As the last example, consider the system (1) of two nonlinear delay integro-differential equations

for $t \in[0,3]$ and $y(t)=\Phi(t)$ for $t \in\left[-\frac{3}{10}, 0\right]$ with

$\Phi_{1}(t)=\frac{1}{4} \sin (t), \Phi_{2}(t)=-\frac{1}{4} \sin (t)$,

$k_{1}(t, s, y(t), y(s))=-y_{1}(t) y_{2}(s)$,

$k_{2}(t, s, y(t), y(s))=e^{s-t} y_{1}(s) y_{2}(t)$,

$f_{1}(t, y(t))=g_{1}(t)+y_{1}(t)\left(1-\frac{1}{3} y_{2}(t)\right)$,

$f_{2}(t, y(t))=g_{2}(t)+y_{2}(t)\left(-2+y_{1}(t)\right)$ where

$g_{1}(t)=\frac{1}{4} \cos (t)-\frac{1}{4} \sin (t)\left(\frac{1}{3}+\frac{1}{2} \sin (t)-\frac{1}{4} \cos (t)+\frac{1}{4} \cos \left(t-\frac{3}{10}\right)\right)$ and 
Table 5: Comparison of the absolute errors for $y_{1}(t)$ of Example 4

\begin{tabular}{lllll}
\hline $\mathrm{t}$ & PLM & ADM & VIM & TCM \\
\hline 0.1 & $1.98 \times 10^{-13}$ & $1.09 \times 10^{-4}$ & $3.15 \times 10^{-4}$ & $1.66 \times 10^{-11}$ \\
0.2 & $3.23 \times 10^{-13}$ & $1.78 \times 10^{-4}$ & $4.27 \times 10^{-4}$ & $6.49 \times 10^{-11}$ \\
0.3 & $3.61 \times 10^{-13}$ & $1.08 \times 10^{-4}$ & $4.72 \times 10^{-4}$ & $1.14 \times 10^{-10}$ \\
0.4 & $3.21 \times 10^{-13}$ & $3.09 \times 10^{-4}$ & $4.85 \times 10^{-4}$ & $1.92 \times 10^{-10}$ \\
0.5 & $2.09 \times 10^{-13}$ & $1.35 \times 10^{-3}$ & $4.74 \times 10^{-4}$ & $3.03 \times 10^{-10}$ \\
0.6 & $3.37 \times 10^{-14}$ & $1.35 \times 10^{-3}$ & $4.45 \times 10^{-4}$ & $4.17 \times 10^{-10}$ \\
0.7 & $1.98 \times 10^{-13}$ & $6.37 \times 10^{-3}$ & $4.36 \times 10^{-4}$ & $4.62 \times 10^{-10}$ \\
0.8 & $4.81 \times 10^{-13}$ & $1.04 \times 10^{-2}$ & $5.35 \times 10^{-4}$ & $5.82 \times 10^{-10}$ \\
0.9 & $8.05 \times 10^{-13}$ & $1.52 \times 10^{-2}$ & $9.10 \times 10^{-4}$ & $6.53 \times 10^{-10}$ \\
1.0 & $1.16 \times 10^{-12}$ & $1.99 \times 10^{-2}$ & $1.82 \times 10^{-3}$ & $7.02 \times 10^{-10}$ \\
& & & &
\end{tabular}

Table 6: Comparison of the absolute errors for $y_{2}(t)$ of Example 4

\begin{tabular}{lllll}
\hline $\mathrm{t}$ & PLM & ADM & VIM & TCM \\
\hline 0.1 & $4.46 \times 10^{-14}$ & $7.58 \times 10^{-6}$ & $3.34 \times 10^{-5}$ & 0 \\
0.2 & $3.74 \times 10^{-14}$ & $1.36 \times 10^{-4}$ & $8.54 \times 10^{-5}$ & $2.50 \times 10^{-14}$ \\
0.3 & $1.10 \times 10^{-14}$ & $7.65 \times 10^{-4}$ & $1.33 \times 10^{-4}$ & $4.32 \times 10^{-13}$ \\
0.4 & $9.03 \times 10^{-14}$ & $2.75 \times 10^{-3}$ & $1.79 \times 10^{-4}$ & $6.36 \times 10^{-12}$ \\
0.5 & $1.89 \times 10^{-13}$ & $7.63 \times 10^{-3}$ & $2.22 \times 10^{-4}$ & $1.03 \times 10^{-11}$ \\
0.6 & $2.98 \times 10^{-13}$ & $1.77 \times 10^{-2}$ & $2.37 \times 10^{-4}$ & $2.13 \times 10^{-11}$ \\
0.7 & $4.06 \times 10^{-13}$ & $3.61 \times 10^{-2}$ & $1.62 \times 10^{-4}$ & $2.00 \times 10^{-11}$ \\
0.8 & $5.02 \times 10^{-13}$ & $6.66 \times 10^{-2}$ & $1.07 \times 10^{-4}$ & $3.29 \times 10^{-11}$ \\
0.9 & $5.76 \times 10^{-13}$ & $1.13 \times 10^{-1}$ & $7.31 \times 10^{-4}$ & $4.45 \times 10^{-11}$ \\
1.0 & $6.18 \times 10^{-13}$ & $1.83 \times 10^{-1}$ & $1.90 \times 10^{-3}$ & $8.10 \times 10^{-11}$ \\
\end{tabular}

$g_{2}(t)=-\frac{1}{4} \cos (t)+\frac{1}{4} \sin (t)\left(-\frac{1}{2}+\frac{3}{8} \sin (t)-\frac{1}{8} \cos (t)+\frac{1}{8} e^{-\frac{3}{10}\left(\cos \left(t-\frac{3}{10}\right)-\sin \left(t-\frac{3}{10}\right)\right)}\right)$.

The exact solution of this system is in the following form $y_{1}(t)=\frac{1}{4} \sin (t), y_{2}(t)=-\frac{1}{4} \sin (t)$.

The absolute errors for $N=3$ and $m=\{5,10\}$ are presented in Table 7 .

Table 7: Absolute errors for $y(t)$ of Example 5

\begin{tabular}{lll|ll}
\hline$t$ & \multicolumn{2}{c|}{$y_{1}(t)$} & \multicolumn{2}{c}{$y_{2}(t)$} \\
& $m=5$ & $m=10$ & $m=5$ & $m=10$ \\
\hline 0.0 & 0.0 & 0.0 & 0.0 & 0.0 \\
0.5 & $7.16 \times 10^{-10}$ & $6.60 \times 10^{-13}$ & $2.63 \times 10^{-10}$ & $4.08 \times 10^{-13}$ \\
1.0 & $5.04 \times 10^{-9}$ & $7.02 \times 10^{-12}$ & $1.51 \times 10^{-9}$ & $6.99 \times 10^{-12}$ \\
1.5 & $1.64 \times 10^{-8}$ & $1.88 \times 10^{-11}$ & $4.71 \times 10^{-9}$ & $5.71 \times 10^{-12}$ \\
2.0 & $3.74 \times 10^{-8}$ & $9.08 \times 10^{-11}$ & $1.01 \times 10^{-8}$ & $1.79 \times 10^{-12}$ \\
2.5 & $6.53 \times 10^{-8}$ & $1.49 \times 10^{-10}$ & $1.55 \times 10^{-8}$ & $4.72 \times 10^{-11}$ \\
3.0 & $9.18 \times 10^{-8}$ & $7.20 \times 10^{-11}$ & $1.72 \times 10^{-8}$ & $1.21 \times 10^{-10}$ \\
\hline
\end{tabular}

Example 6 ([25]) Consider the system (1) of two nonlinear delay integrodifferential equations with 
$\Phi_{1}(t)=t^{2}-t, \Phi_{2}(t)=-e^{-3 t}$ for $t \in\left[-\frac{1}{4}, 0\right]$,

$k_{1}(t, s, y(t), y(s))=(s-t) y_{1}(t) y_{2}(s)$,

$k_{2}(t, s, y(t), y(s))=(t-s+1) y_{1}(s) y_{2}(t)$,

$f_{1}(t, y(t))=2 t-1-\left(t^{2}-t\right)\left(1+\frac{11}{18} e^{-3 t}-\frac{1}{36} e^{\frac{3}{4}-3 t}\right)+y_{1}(t)\left(1-\frac{1}{2} y_{2}(t)\right)$,

$f_{2}(t, y(t))=\frac{1}{3072} e^{-3 t}\left(10080 t^{2}-10304 t+6275\right)+y_{2}(t)\left(-1+3 y_{1}(t)\right)$.

The exact solution of this system is in the following form $y_{1}(t)=t^{2}-t, y_{2}(t)=$ $-e^{-3 t}$.

The absolute errors of Taylor Collocation Method (TCM) for $m=8, N=8$ are compared with the absolute error of the Variational Iteration Method (VIM), Adomian Decomposition Method (ADM) and Pseudospectral Legendre Method (PLM) given in [25] in Table 8 and Table 9.

Table 8: Comparison of the absolute errors for $y_{1}(t)$ of Example 6

\begin{tabular}{lllll}
\hline $\mathrm{t}$ & PLM & ADM & VIM & TCM \\
\hline 0.1 & $7.99 \times 10^{-4}$ & $2.48 \times 10^{-5}$ & $3.59 \times 10^{-7}$ & $1.51 \times 10^{-12}$ \\
0.2 & $1.48 \times 10^{-3}$ & $9.91 \times 10^{-5}$ & $2.66 \times 10^{-7}$ & $7.21 \times 10^{-12}$ \\
0.3 & $2.05 \times 10^{-3}$ & $2.16 \times 10^{-4}$ & $4.66 \times 10^{-7}$ & $3.86 \times 10^{-12}$ \\
0.4 & $2.52 \times 10^{-3}$ & $3.80 \times 10^{-4}$ & $1.64 \times 10^{-5}$ & $8.68 \times 10^{-12}$ \\
0.5 & $2.91 \times 10^{-3}$ & $6.11 \times 10^{-4}$ & $6.93 \times 10^{-5}$ & $2.48 \times 10^{-11}$ \\
0.6 & $3.22 \times 10^{-3}$ & $9.58 \times 10^{-4}$ & $1.73 \times 10^{-4}$ & $2.94 \times 10^{-11}$ \\
0.7 & $3.47 \times 10^{-3}$ & $1.50 \times 10^{-3}$ & $3.23 \times 10^{-4}$ & $3.78 \times 10^{-11}$ \\
0.8 & $3.67 \times 10^{-3}$ & $2.40 \times 10^{-3}$ & $4.92 \times 10^{-4}$ & $7.34 \times 10^{-12}$ \\
0.9 & $3.82 \times 10^{-3}$ & $3.81 \times 10^{-3}$ & $6.41 \times 10^{-4}$ & $4.08 \times 10^{-11}$ \\
1.0 & $3.95 \times 10^{-3}$ & $5.92 \times 10^{-3}$ & $7.37 \times 10^{-4}$ & $9.12 \times 10^{-11}$ \\
\hline
\end{tabular}

Table 9: Comparison of the absolute errors for $y_{2}(t)$ of Example 6

\begin{tabular}{lllll}
\hline $\mathrm{t}$ & PLM & ADM & VIM & TCM \\
\hline 0.1 & $3.61 \times 10^{-2}$ & $4.87 \times 10^{-5}$ & $1.09 \times 10^{-5}$ & $2.88 \times 10^{-12}$ \\
0.2 & $4.53 \times 10^{-2}$ & $4.44 \times 10^{-5}$ & $1.55 \times 10^{-5}$ & $4.44 \times 10^{-12}$ \\
0.3 & $4.06 \times 10^{-2}$ & $1.62 \times 10^{-4}$ & $8.22 \times 10^{-6}$ & $3.35 \times 10^{-11}$ \\
0.4 & $3.06 \times 10^{-2}$ & $8.70 \times 10^{-4}$ & $9.26 \times 10^{-5}$ & $5.90 \times 10^{-11}$ \\
0.5 & $2.04 \times 10^{-2}$ & $2.49 \times 10^{-3}$ & $3.95 \times 10^{-4}$ & $3.91 \times 10^{-11}$ \\
0.6 & $1.30 \times 10^{-2}$ & $5.43 \times 10^{-3}$ & $9.37 \times 10^{-4}$ & $4.34 \times 10^{-11}$ \\
0.7 & $9.06 \times 10^{-3}$ & $9.83 \times 10^{-3}$ & $1.62 \times 10^{-3}$ & $5.66 \times 10^{-11}$ \\
0.8 & $8.19 \times 10^{-3}$ & $1.53 \times 10^{-2}$ & $2.26 \times 10^{-3}$ & $2.15 \times 10^{-10}$ \\
0.9 & $8.96 \times 10^{-3}$ & $2.10 \times 10^{-2}$ & $2.63 \times 10^{-3}$ & $7.67 \times 10^{-11}$ \\
1.0 & $9.14 \times 10^{-3}$ & $2.54 \times 10^{-2}$ & $2.57 \times 10^{-3}$ & $2.61 \times 10^{-10}$ \\
\hline
\end{tabular}


Example 7 Consider the following five-dimensional nonlinear system:

$$
\left\{\begin{array}{l}
y_{1}^{\prime}(t)=g_{1}(t)-y_{1}(t) y_{3}(t)-y_{1}(t) \\
y_{2}^{\prime}(t)=y_{1}(t)-y_{5}(t)-y_{2}(t) \\
y_{3}^{\prime}(t)=y_{3}(t)\left(2-y_{3}(t)\right)-y_{4}(t) y_{5}(t) \\
y_{4}^{\prime}(t)=g_{2}(t)+\int_{t-\frac{1}{4}}^{t}\left(s-y_{3}(t)\right) d s-\frac{1}{4} y_{4}(t) \\
y_{5}^{\prime}(t)=g_{3}(t)+\int_{t-\frac{1}{4}}^{t} s y_{3}(t) d s-\frac{1}{2} y_{5}(t)
\end{array}, t \in[0,2]\right.
$$

$g_{1}(t), g_{2}(t), g_{3}(t)$ are chosen so that the exact solution is $y(t)=\left(-e^{-2 t}+\cos (t), e^{-2 t}, t^{2}+1,1-2 t-t^{4}-\cos (t), \cos (t)\right)$ and $y(t)=\Phi(t)$ for $t \in\left[-\frac{1}{4}, 0\right]$. The maximum errors that have been obtained for system (37) for $(N, m)=\{(3,3),(4,4),(5,5),(6,6)\}$ are presented in Table 10.

Table 10: The maximum errors of system (37)

\begin{tabular}{lllll}
\hline$(N, m)$ & $(3,3)$ & $(4,4)$ & $(5,5)$ & $(6,6)$ \\
\hline$y_{1}(t)$ & $4.83 \times 10^{-5}$ & $1.96 \times 10^{-7}$ & $1.25 \times 10^{-9}$ & $3.68 \times 10^{-10}$ \\
$y_{2}(t)$ & $3.83 \times 10^{-5}$ & $2.15 \times 10^{-7}$ & $8.87 \times 10^{-10}$ & $7.26 \times 10^{-10}$ \\
$y_{3}(t)$ & $2.61 \times 10^{-4}$ & $7.12 \times 10^{-9}$ & $1.19 \times 10^{-9}$ & $1.12 \times 10^{-9}$ \\
$y_{4}(t)$ & $9.37 \times 10^{-4}$ & $1.32 \times 10^{-7}$ & $2.01 \times 10^{-9}$ & $7.41 \times 10^{-10}$ \\
$y_{5}(t)$ & $7.84 \times 10^{-5}$ & $9.89 \times 10^{-8}$ & $4.01 \times 10^{-10}$ & $1.06 \times 10^{-10}$ \\
\hline
\end{tabular}

Example 8 ([18]) In this example, we found a numerical solution for the SEIR model based on the four nonlinear ordinary differential equations describing the COVID-19 epidemic in China. It has four elements which are S (susceptible), E (exposed), I (infectious) and R (recovered) and can be represented as follows:

$$
\left\{\begin{array}{l}
\frac{d S}{d t}(t)=-\beta \frac{S(t)}{N} I(t)-\frac{Z}{N} S(t)+\rho_{I}+\rho_{E}-\left(\frac{\rho_{I}}{N}+\frac{\rho_{E}}{N}\right) S(t)+v N(t)-\mu S(t) \\
\frac{d E}{d t}(t)=\beta \frac{S(t)}{N} I(t)+\frac{Z}{N} S(t)-\alpha E(t)-\left(\frac{\rho_{I}}{N}+\frac{\rho_{E}}{N}\right) E(t)-\mu E(t)-\sigma E(t) \\
\frac{d I}{d t}(t)=\alpha E(t)-\gamma I(t)-\left(\frac{\rho_{I}}{N}+\frac{\rho_{E}}{N}\right) I(t)-\mu I(t) \\
\frac{d R}{d t}(t)=\gamma I(t)-\mu R(t)+\sigma E(t) .
\end{array}\right.
$$

For $t \in[0,20]$, with initial conditions $S(0)=2500, E(0)=1, I(0)=1, R(0)=0$, $N(t)=S(t)+E(t)+I(t)+R(t)=2502$ and parameters $\beta=0.8, \alpha=0.75, \sigma=0.1$, $\gamma=0.05, v=0.009 / N, \mu=0.01, Z=0.001, \rho_{I}=0.15, \rho_{E}=0.15, \rho_{I}=0.01$, $\rho_{E}=0.03$.

We used Taylor Collocation Method (TCM) for $h=\frac{1}{4},(N, m)=(80,6)$ and 
compared the approximate solution obtained with the approximate solution of the variational iteration method (VIM) and the differential transformation method (DTM) given in [18] in table (11). For more information about the model refer to [9].

Table 11: Comparison of approximate solutions of the SEIR model (38) for compartment S, E, I and R using VIM, DTM and TCM.

\begin{tabular}{lccc}
\hline $\mathrm{t}$ & DTM & VIM & TCM \\
\hline 0 & 2500 & 2500 & 2500 \\
2 & 2448 & 2448 & 2448 \\
4 & 2396 & 2396 & 2394 \\
6 & 2345 & 2345 & 2334 \\
8 & 2294 & 2293 & 2260 \\
10 & 2244 & 2243 & 2157 \\
12 & 2197 & 2195 & 2000 \\
14 & 2152 & 2149 & 1754 \\
16 & 2111 & 2105 & 1398 \\
18 & 2075 & 2065 & 967 \\
20 & 2043 & 2028 & 567 \\
\hline \multicolumn{4}{c}{ Compartment S } \\
\hline $\mathrm{t}$ & DTM & VIM & TCM \\
\hline 0 & 1 & 1 & 1 \\
2 & 3 & 3 & 3 \\
4 & 8 & 8 & 6 \\
6 & 20 & 19 & 13 \\
8 & 42 & 40 & 28 \\
10 & 79 & 74 & 59 \\
12 & 133 & 124 & 122 \\
14 & 209 & 193 & 237 \\
16 & 309 & 283 & 425 \\
18 & 438 & 396 & 675 \\
20 & 599 & 537 & 923 \\
\hline & Compartment I \\
& &
\end{tabular}

\begin{tabular}{|c|c|c|c|}
\hline $\mathrm{t}$ & DTM & VIM & $\mathrm{TCM}$ \\
\hline 0 & 1 & 1 & 1 \\
\hline 2 & 1 & 1 & 2 \\
\hline 4 & -1 & -1 & 4 \\
\hline 6 & -10 & -9 & 8 \\
\hline 8 & -31 & -29 & 17 \\
\hline 10 & -69 & -64 & 34 \\
\hline 12 & -128 & -117 & 66 \\
\hline 14 & -214 & -194 & 119 \\
\hline 16 & -331 & -296 & 184 \\
\hline 18 & -484 & -429 & 230 \\
\hline 20 & -677 & -594 & 221 \\
\hline \multicolumn{4}{|c|}{ Compartment E } \\
\hline $\mathrm{t}$ & DTM & VIM & TCM \\
\hline 0 & 0 & 0 & 0 \\
\hline 2 & 0 & 0 & 0 \\
\hline 4 & 1 & 1 & 1 \\
\hline 6 & 3 & 3 & 3 \\
\hline 8 & 7 & 7 & 7 \\
\hline 10 & 12 & 12 & 16 \\
\hline 12 & 20 & 19 & 34 \\
\hline 14 & 30 & 28 & 69 \\
\hline 16 & 43 & 40 & 129 \\
\hline 18 & 60 & 56 & 223 \\
\hline 20 & 82 & 74 & 343 \\
\hline
\end{tabular}




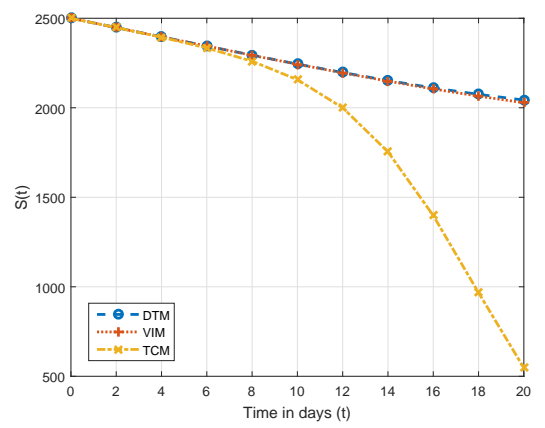

(a) Compartment $\mathrm{S}$

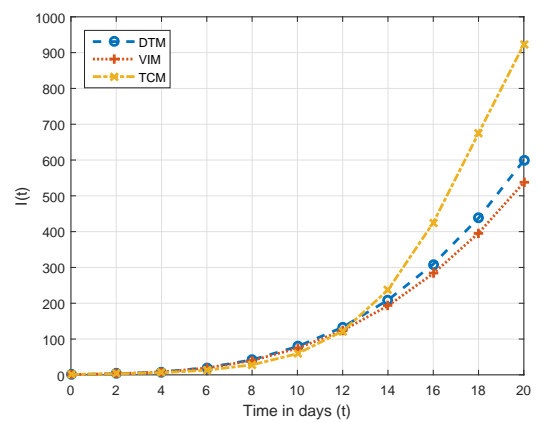

(c) Compartment I

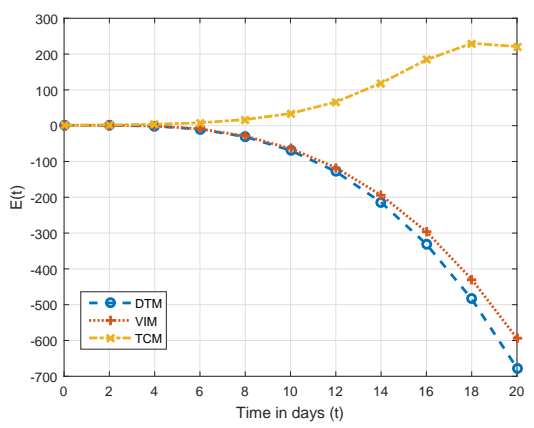

(b) Compartment E

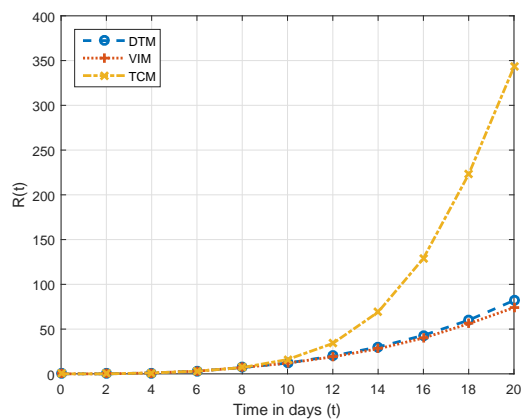

(d) Compartment $\mathrm{R}$

Fig. 1: Comparison of approximate solutions of the SEIR model (38) for compartment S, E, I and R using VIM, DTM and TCM.

Example 9 ([22]) In this example, we found a numerical solution for the SEIRU model based on a DDEs (delay differential equations), describing the COVID19 epidemic in China. This system has five elements which are:

$S$ is the number of individuals susceptible to infection, $E$ is the number of asymptomatic noninfectious individuals, $I$ is the number of asymptomatic but infectious individuals, $R$ is the number of reported symptomatic infectious individuals, and $U$ is the number of unreported symptomatic infectious individuals, can be represented in the folowing system:

$$
\left\{\begin{array}{l}
\frac{d S}{d t}(t)=-D(t) S(t)(I(t)+U(t)) \\
\frac{d E}{d t}(t)=D(t) S(t)(I(t)+U(t))-D(t-\tau) S(t-\tau)(I(t-\tau)+U(t-\tau)) \\
\frac{d I}{d t}(t)=D(t-\tau) S(t-\tau)(I(t-\tau)+U(t-\tau))-v I(t) \\
\frac{d R}{d t}(t)=v_{1} I(t)-\eta R(t) \\
\frac{d U}{d t}(t)=v_{2} I(t)-\eta U(t) .
\end{array}\right.
$$


For $t \in[0,72]=\left[\begin{array}{lll}\text { January } & 6, \text { March } 18\end{array}\right]$. This system is supplemented by initial fonctions for $t \in[-\tau, 0]$

$$
\left\{\begin{aligned}
S(t) & =1400050000 \\
E(t) & =\tau(0.3762+v) I(t) \\
I(t) & =\frac{0.3762}{0.8 v} \exp (0.3762 t) \\
R(t) & =0 \\
U(t) & =\frac{0.2 v}{\eta+0.3762} I(t)
\end{aligned}\right.
$$

The time-dependent transmission rate parameter $D$ is

$$
D(t)=\left\{\begin{array}{c}
D_{0}=\left(\frac{0.3762+v}{1400050000}\right)\left(\frac{\eta+0.3762}{v_{2}+\eta+0.3762}\right) \exp (0.3762 \tau) \quad 0 \leq t \leq N_{0} \\
D_{0} \exp \left(-\mu\left(t-N_{0}\right)\right) \quad N_{0}<t
\end{array}\right.
$$

Where the day $N_{0}=19$ (January 25) corresponds to the day when the public measures take effect, $\mu=0.62$ is the rate at which they take effect, it is chosen so that the simulations align with the cumulative reported case data and the parameters $\tau=-\frac{1}{4}, v=\frac{1}{7}, v_{1}=\frac{0.8}{7}, v_{2}=\frac{0.2}{7}, \eta=\frac{1}{7}$. For more information about the model refer to $[22]$.

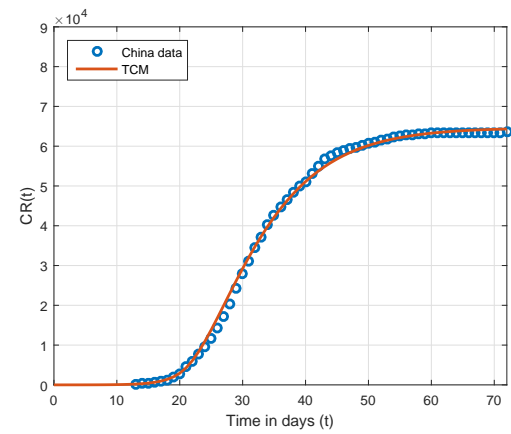

(a) Reported case data and $t \rightarrow C R(t)$.

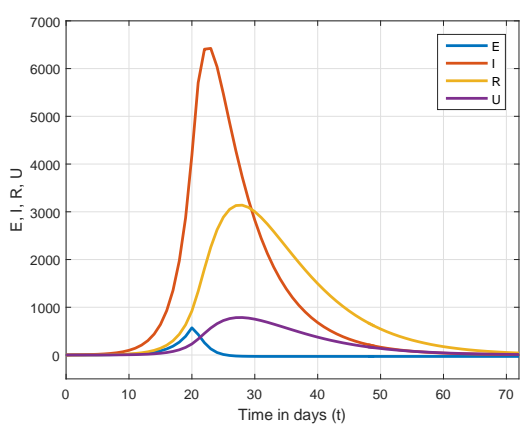

(b) Compartment E, I, R and U.

Fig. 2: Approximate solutions of the SEIRU model (39) for compartment E, I,R and U using TCM.

\section{Conclusion}

In this paper, we have proposed a collocation method based on the use of Taylor polynomials to approximate the solution of the general system of nonlinear delay integro-differential equations (1) in the spline space $S_{m}^{(0)}\left(\Pi_{N}\right)$. We have shown that the numerical solution is convergent. This method is easy to implement and the coefficients of the approximation solution are determined by 
Table 12: Comparison of cumulative daily reported case data [22] with approximate solutions of the SEIRU model (39) for the cumulative number of reported symptomatic infectious cases $(t \rightarrow C R(t))$ using TCM

\begin{tabular}{|c|c|c|c|c|c|c|c|c|}
\hline \multicolumn{9}{|c|}{ January } \\
\hline Day & 16 & 17 & 18 & 19 & 20 & 21 & 22 & 23 \\
\hline $\mathrm{CR}($ China data $)$ & & & & 198 & 291 & 440 & 571 & 830 \\
\hline $\mathrm{CR}(\mathrm{TCM})$ & 63 & 94 & 139 & 205 & 302 & 442 & 647 & 945 \\
\hline Day & 24 & 25 & 26 & 27 & 28 & 29 & 30 & 31 \\
\hline $\mathrm{CR}$ (China data) & 1287 & 1975 & 2744 & 4515 & 5974 & 7711 & 9692 & 11791 \\
\hline $\mathrm{CR}(\mathrm{TCM})$ & 1379 & 2011 & 2933 & 4266 & 6075 & 8331 & 10952 & 13838 \\
\hline \multicolumn{9}{|c|}{ February } \\
\hline Day & 1 & 2 & 3 & 4 & 5 & 6 & 7 & 8 \\
\hline $\mathrm{CR}$ (China data) & 14380 & 17205 & 20438 & 24324 & 28018 & 31161 & 34546 & 37198 \\
\hline $\mathrm{CR}(\mathrm{TCM})$ & 16889 & 20019 & 23158 & 26250 & 29253 & 32136 & 34878 & 37466 \\
\hline Day & 9 & 10 & 11 & 12 & 13 & 14 & 15 & 16 \\
\hline CR(China data) & 40171 & 42638 & 44653 & 46472 & 48467 & 49970 & 51091 & 53139 \\
\hline $\mathrm{CR}(\mathrm{TCM})$ & 39891 & 42153 & 44251 & 46188 & 47971 & 49606 & 51101 & 52464 \\
\hline Day & 17 & 18 & 19 & 20 & 21 & 22 & 23 & 24 \\
\hline $\mathrm{CR}($ China data $)$ & 55027 & 56776 & 57593 & 58482 & 58879 & 59527 & 59741 & 60249 \\
\hline $\mathrm{CR}(\mathrm{TCM})$ & 53704 & 54829 & 55849 & 56770 & 57602 & 58352 & 59026 & 59633 \\
\hline Day & 25 & 26 & 27 & 28 & 29 & & & \\
\hline $\mathrm{CR}$ (China data) & 60655 & 61088 & 61415 & 61806 & 62415 & & & \\
\hline $\mathrm{CR}(\mathrm{TCM})$ & 60177 & 60665 & 61101 & 61492 & 61841 & & & \\
\hline \multicolumn{9}{|c|}{ March } \\
\hline Day & 1 & 2 & 3 & 4 & 5 & 6 & 7 & 8 \\
\hline $\mathrm{CR}$ (China data) & 62617 & 62742 & 62861 & 63000 & 63143 & 63242 & 63286 & 63326 \\
\hline $\mathrm{CR}(\mathrm{TCM})$ & 62153 & 62432 & 62680 & 62901 & 63097 & 63272 & 63428 & 63566 \\
\hline Day & 9 & 10 & 11 & 12 & 13 & 14 & 15 & 16 \\
\hline $\mathrm{CR}$ (China data) & 63345 & 63369 & 63384 & 63404 & 63415 & 63435 & 63451 & 63472 \\
\hline $\mathrm{CR}(\mathrm{TCM})$ & 63688 & 63797 & 63894 & 63979 & 64055 & 64122 & 64182 & 64235 \\
\hline Day & 17 & 18 & 19 & 20 & 21 & 22 & 23 & 24 \\
\hline $\mathrm{CR}($ China data $)$ & 63485 & 63519 & & & & & & \\
\hline $\mathrm{CR}(\mathrm{TCM})$ & 64281 & 64322 & & & & & & \\
\hline
\end{tabular}

iterative formulas without the need to solve any system of algebraic equations. The numerical examples which were introduced have shown that the method is convergent with a good accuracy.

Acknowledgements This work is supported by the Directorate General for Scientific Research and Technological Development (DGRSDT), Ministry of Higher Education and Scientific Research, Algeria. 


\section{References}

1. Abbasbandy, S., Taati, A.: Numerical solution of the system of nonlinear Volterra integrodifferential equations with nonlinear differential part by the operational Tau method and error estimation. Journal of Computational and Applied Mathematics. 231(1), 106-113 (2009)

2. Abdi, A., Berrut, J.P, Hosseini, S.A.: The linear barycentric rational method for a class of delay Volterra integro-differential equations. Journal of Scientific Computing. 75(3), 1757-1775 (2018)

3. Adomian, G.: A review of the decomposition method and some recent results for nonlinear equations. Math. Comput. Model. 13(7), 17-43 (1990)

4. Agarwal, R.P.: Difference equations and inequalities: theory, methods, and applications. CRC Press. (2000)

5. Arikoglu, A., Ozkol, I.: Solutions of integral and integro-differential equation systems by using differential transform method. Computers and Mathematics with Applications. 56(9), 2411-2417 (2008)

6. Bellour, A., Bousselsal, M.: Numerical solution of delay integro-differential equations by using Taylor collocation method. Math. Meth. Appl. Sci. 37, 1491-1506 (2014)

7. Bellour, A., Bousselsal, M.: A Taylor collocation method for solving delay integral equations. Numerical Algorithms. 65, 843-857 (2014)

8. Biazar, J., Ghazvini, H., Eslami, M.: He's homotopy perturbation method for systems of integro-differential equations. Chaos, Solitons and Fractals. 39(3), 1253-1258 (2009)

9. Biswas, M.H.A., Paiva, L.T., De Pinho, M.D.R.: A SEIR model for control of infectious diseases with constraints. Mathematical Biosciences and Engineering. 11(4), 761 (2014)

10. Brunner, H.: Collocation methods for Volterra integral and related functional differential equations. Cambridge university press, Cambridge (2004)

11. Burton, T.A.: Volterra Integral and Differential Equations. Academic Press, New York (1983)

12. Cushing, J.M.: Integrodifferential equations and delay models in population dynamics (Vol. 20). Springer Science and Business Media (2013)

13. Darania, P.: Multistep collocation method for nonlinear delay integral equations. Sahand Commun. Math. Anal. (SCMA). 3, 47-65 (2016)

14. Darania, P., Pishbin, S.: High-order collocation methods for nonlinear delay integral equation. J. Comput. Appl. 326, 284-295 (2017)

15. Fazeli, S., Hojjati, G.: Numerical solution of Volterra integro-differential equations by superimplicit multistep collocation methods. Numer. Algorithms. 68, 741-768 (2015)

16. Gülsu, M., Sezer, M.: Taylor collocation method for solution of systems of high-order linear Fredholm Volterra integro-differential equations. Int. J. Comput. 83, 429-448 (2006)

17. Gu, Z.: Chebyshev spectral collocation method for system of nonlinear Volterra integral equations. Numerical Algorithms. 83(1), 243-263 (2020)

18. Harir, A., Melliani, S., El Harfi, H., Chadli, L.S.: Variational Iteration Method and Differential Transformation Method for Solving the SEIR Epidemic Model. Int. J. Differ. Equ. vol 2020 (2020)

19. Hairer, E., Lubich, C., N $\phi$ rsett, S.P.: Order of convergence of one-step methods for Volterra integral equations of the second kind. SIAM J. Numer. Anal. 20, 569-579 (1983)

20. He, J.H.: Approximate solution of nonlinear differential equations with convolution product nonlinearities. Comput. Methods Appl. Mech. Eng. 167, 69-73 (1998)

21. Jerri, A.J.: Introduction to Integral Equations with Applications. John Wiley and Sons, INC (1999)

22. Liu, Z., Magal, P., Seydi, O., Webb, G.: A COVID-19 epidemic model with latency period. Infectious Disease Modelling. 5, 323-337 (2020)

23. Rihan, F. A., Doha, E.H., Hassan, M., Kamel, N.M.: Numerical treatments for volterra delay integro-differential equations. Comput. Methods Appl. Math. 9(3), 292-308 (2009)

24. Saberi-Nadjafi, J., Tamamgar, M.: The variational iteration method: a highly promising method for solving the system of integro-differential equations. Computers and Mathematics with Applications. 56(2), 346-351 (2008)

25. Shakeri, F., Dehghan, M.: Solution of a model describing biological species living together using the variational iteration method. Math. Comput. Model. 48, 685-699 (2008) 
26. Shakourifar, M., Enright, W.: Superconvergent interpolants for collocation methods applied to Volterra integro-differential equations with delay. BIT Numerical Mathematics. 52(3), 725-740 (2012)

27. Volterra, V.: Variazioni e fluttuazioni del numero d'individui in specie animali conviventi (p. 142). C. Ferrari. (1927)

28. Wang, K., Wang, Q.: Taylor collocation method and convergence analysis for the Volterra Fredholm integral equations. J. Comput. Appl. 260, 294-300 (2014)

29. Wazwaz, A.M.: Linear and nonlinear integral equations (Vol. 639). Berlin, Springer. (2011)

30. Yüzbaşı, S.., Sezer, M.: An exponential approach for the system of nonlinear delay integro-differential equations describing biological species living together. Neural Computing and Applications. 27(3), 769-779 (2016) 
Figures

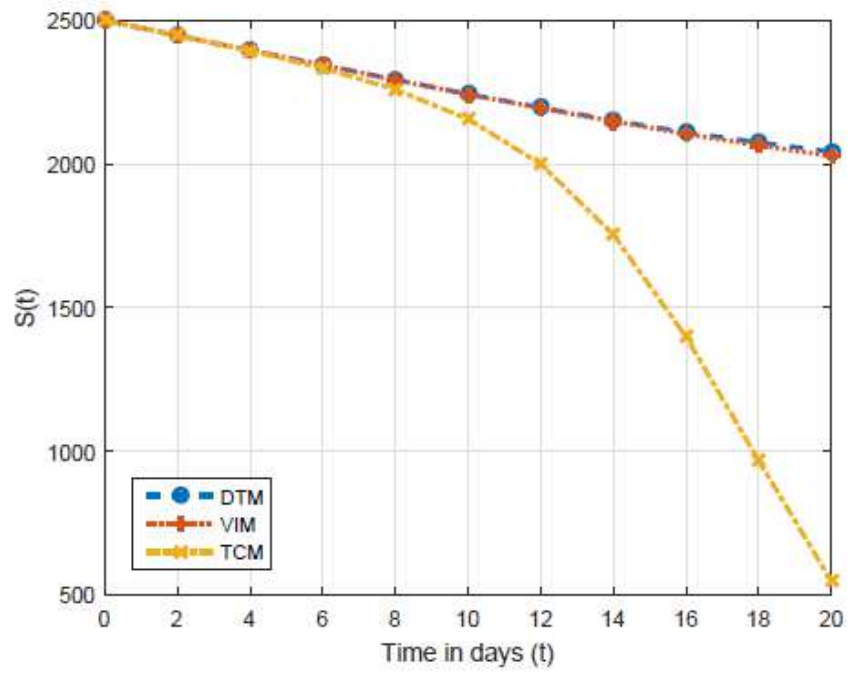

(a) Compartment S .

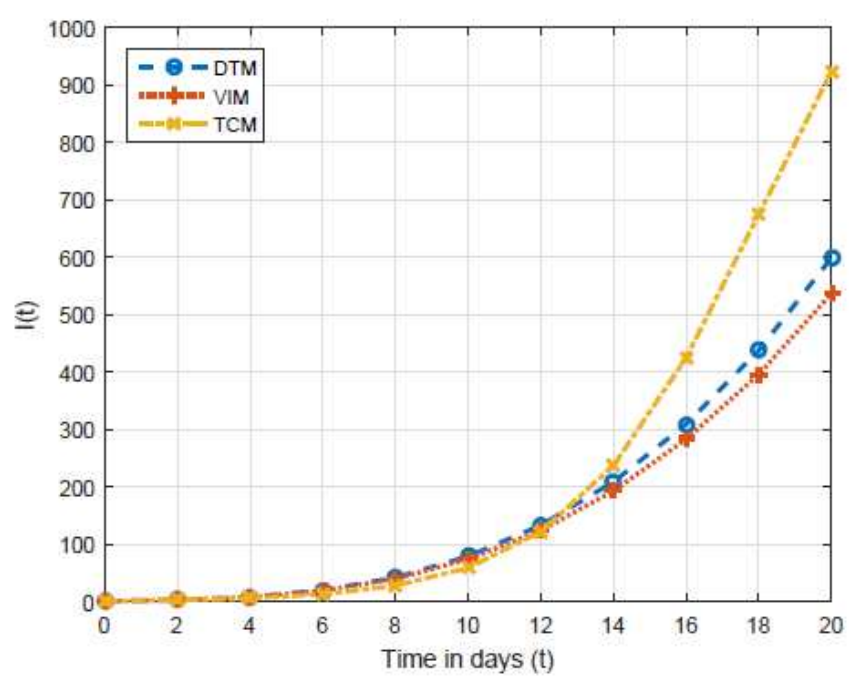

(c) Compartment I .

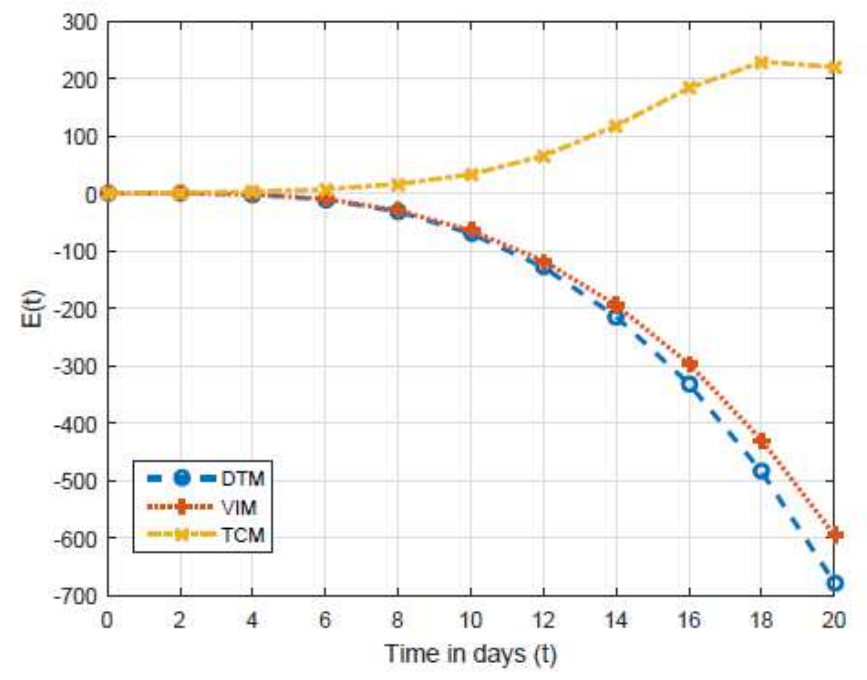

(b) Compartment E .

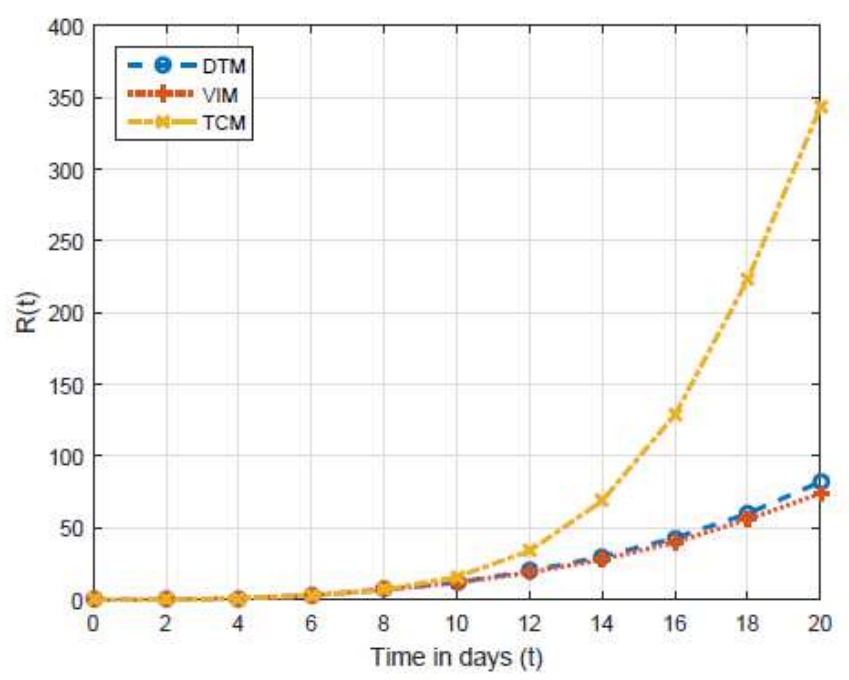

(d) Compartment R .

\section{Figure 1}

Comparison of approximate solutions of the SEIR model (38) for compartment S, E, I and R using VIM, DTM and TCM. 


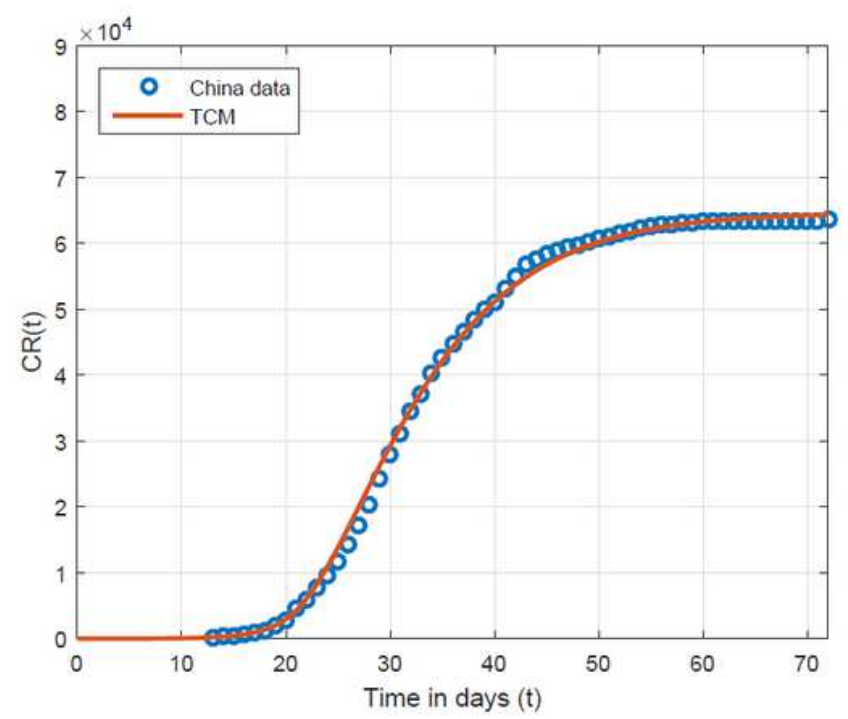

(a) Reported case data and $t \rightarrow C R(t)$.

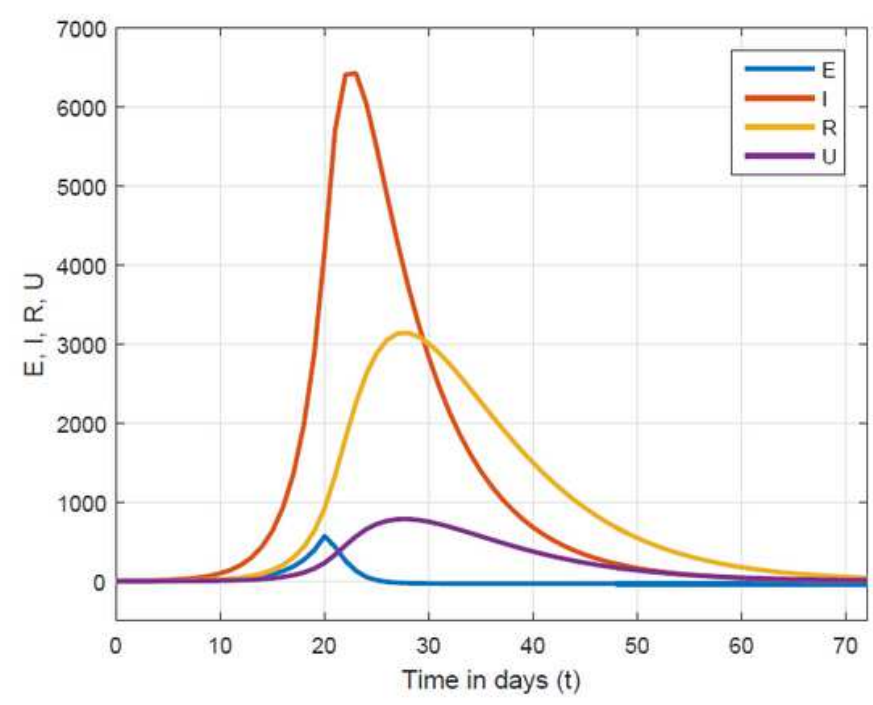

(b) Compartment E, I, R and U.

Figure 2

Approximate solutions of the SEIRU model (39) for compartment E, I,R and U using TCM. 